\title{
DNS of Receptivity to Freestream Acoustic Noise in Hypersonic Flow over a Generic Forebody
}

\author{
Adriano Cerminara* \\ University of Southampton, Southampton, SO17 1BJ, United Kingdom \\ Antoine Durant ${ }^{\dagger}$ \\ MBDA France, 92350 Le Plessis-Robinson, France \\ Thierry André \\ MBDA France, 18020 Bourges, France \\ Neil D. Sandham ${ }^{\S}$ \\ University of Southampton, SO17 1BJ Southampton, United Kingdom \\ Nigel J. Taylor $\llbracket$ \\ MBDA UK Ltd, Filton, BS34 7QW, United Kingdom
}

\begin{abstract}
Direct numerical simulations (DNS) of the Navier-Stokes equations have been performed to investigate the receptivity and breakdown mechanisms in a Mach 6 flow over a generic forebody geometry with freestream acoustic disturbances. The simulations are based on transition experiments carried out in April 2015 in the Boeing/AFOSR Mach 6 facility at Purdue University. A three-dimensional model for both fast and slow freestream acoustic waves with multiple frequencies and spanwise wavenumbers has been adopted in the numerical simulations, for which high-amplitude disturbances have been considered in order to simulate noisy wind tunnel conditions. The numerical results reveal similarities in comparison to the experimental observations, especially when slow acoustic waves are considered as freestream disturbances. In particular, slow acoustic waves have been found to induce the breakdown process via crossflow instabilities located in the off-centerline region, with formation of streamwise streaks. Fast acoustic waves, in contrast, appear more efficient in inducing earlier nonlinear growth through destabilisation of the boundary layer along the symmetry plane of the body.
\end{abstract}

* Research Fellow, Member AIAA

$\dagger$ Aerospace Engineer. Aerodynamics and Performances

$¥$ Aerospace Engineer

§Professor of Aerospace Engineering

ॠCapability Leader Aerodynamic Tools and Methods, AIAA Associate Fellow

$$
1 \text { of } 31
$$




\section{Nomenclature}

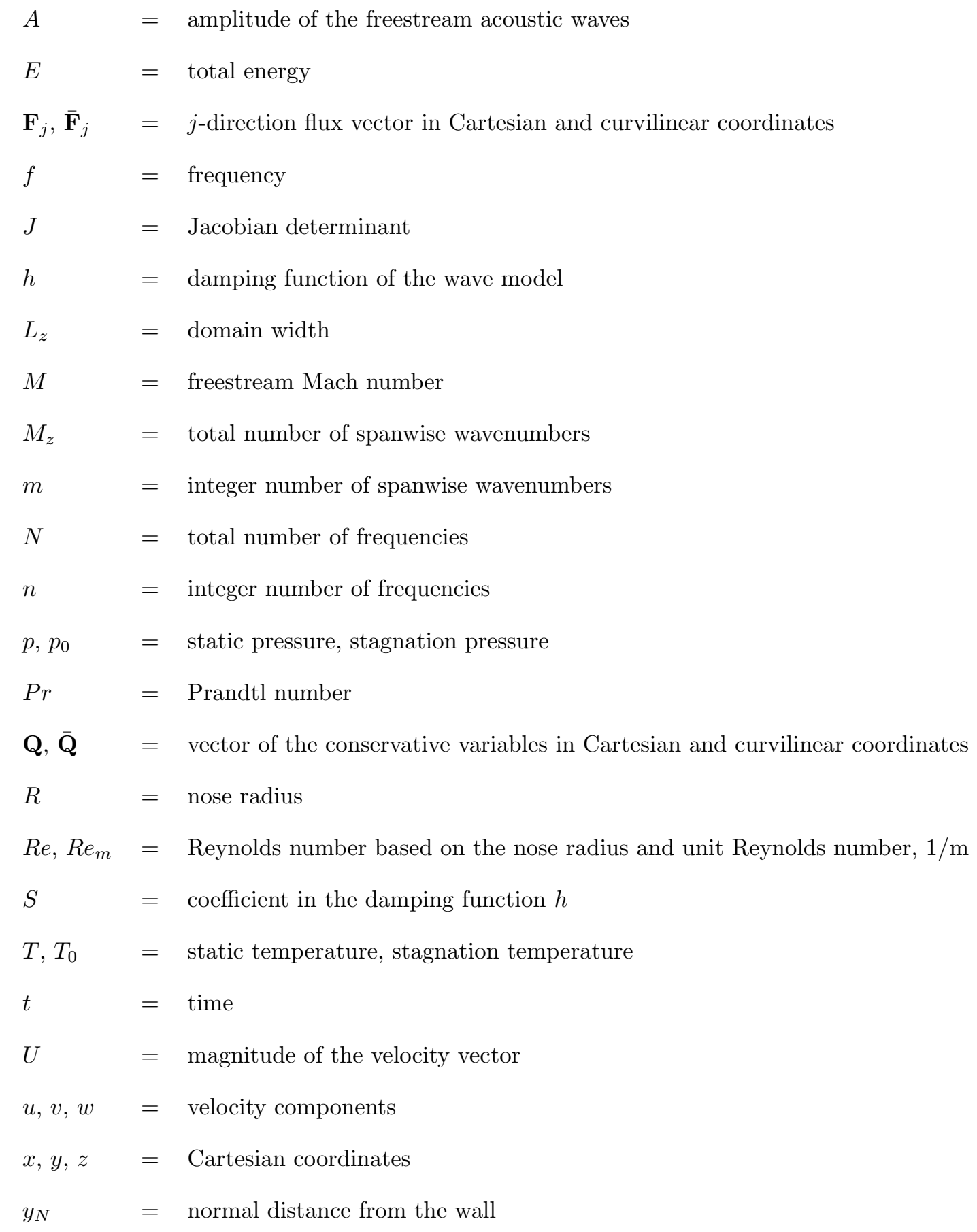




\begin{tabular}{|c|c|c|}
\hline$\alpha$ & $=$ & streamwise wavenumber \\
\hline$\beta$ & $=$ & spanwise wavenumber \\
\hline$\gamma$ & $=$ & ratio of the specific heats \\
\hline$\delta_{i j}$ & $=$ & Kronecker delta \\
\hline$\theta$ & $=$ & inclination angle of the acoustic waves in the $x z$ plane \\
\hline$\mu$ & $=$ & dynamic viscosity \\
\hline$\xi_{j}$ & $=$ & curvilinear coordinates \\
\hline$\rho$ & $=$ & density \\
\hline$\tau_{i j}$ & $=$ & viscous stresses \\
\hline$\tau$ & $=$ & leading edge tangential direction in the $x z$ plane \\
\hline$\psi$ & $=$ & phase angle of the acoustic waves in the streamwise direction \\
\hline$\omega$ & $=$ & angular frequency \\
\hline \multicolumn{3}{|c|}{ Subscripts } \\
\hline$m$ & $=$ & $m^{t h}$ spanwise wavenumber \\
\hline$n$ & $=$ & $n^{t h}$ frequency \\
\hline$w$ & $=$ & value at the wall \\
\hline$x, y, z$ & $=$ & cartesian coordinates \\
\hline$\infty$ & $=$ & freestream value \\
\hline \multicolumn{3}{|c|}{ Superscripts } \\
\hline * & $=$ & dimensional value \\
\hline 1 & $=$ & fluctuating value \\
\hline
\end{tabular}

\section{Introduction}

Transition to turbulence plays a crucial role in determining the heat-transfer rates on the surface of hypersonic vehicles, which makes it necessary to understand the physical mechanisms responsible for transition, in order to allow accurate prediction of the transition location. The main path to transition, in the case of linear disturbances, can be summarized in three fundamental steps: i) receptivity, ii) modal growth of the instabilities and c) nonlinear instability and final breakdown to turbulence.

Receptivity is the internalization process of the external disturbances into the boundary layer in the form of instability modes, and determines the initial conditions for the downstream linear growth of the unstable modes. In the case of high-amplitude disturbances, nonlinearities can become predominant in the 
receptivity process, which would lead directly to nonlinear growth and rapid transition to turbulence. In some particular conditions, an intermediate route to transition (between a linear modal and a fully nonlinear process) is possible, i.e. transient growth, consisting of a non-modal growth of linear instabilities, as in the case of lift-up-induced streamwise streaks in conjunction with secondary instabilities and consequent nonlinear breakdown [1, 2].

The body leading edge is a highly-receptive zone, due to the non-parallel effects and the related shortscale streamwise variations of the mean flow, which, in turn, cause a wavelength-conversion process from the scale of the external forcing to that of the induced boundary-layer disturbances 3 . At hypersonic Mach numbers, however, the small difference in phase speed between the forcing waves and the boundary-layer dominant modes can lead to a direct excitation of these modes via a resonance mechanism at the leading edge [4, 5, 6, 7, without the need of a wavelength-conversion mechanism. By applying Fedorov's notation [5], the internal mode generated at the leading edge through the receptivity to fast acoustic waves is called the fast mode, or mode $\mathrm{F}$, while the mode associated to the slow acoustic waves is known as the slow mode, or mode $\mathrm{S}$, which is the mode pertaining to the class of the unstable boundary-layer modes. In hypersonic boundary layers, different unstable modes can coexist, including the first mode, corresponding to the Tollmien-Schlichting (TS) waves, and the higher-frequency (second, third, etc.) 'Mack' modes [8]. Among the high-frequency modes, the second mode was found to play a crucial role in the transition process by the experiment of Stetson et al. $[9$ for a Mach 8 flow over a cone, and is, in general, known to be the dominant instability mode in two-dimensional (2D) hypersonic boundary layers [5, 10]. The receptivity to fast and slow acoustic waves has been numerically studied by several authors [6, 11, 12, 13, 14, 15], whose results agree, in general, in recognizing a major role played by the slow acoustic waves (in the linear case) in the maximum amplitude reached by the unstable second mode and, hence, in the transition process, compared to fast acoustic waves. However, recent numerical studies [16, 17] found that for high-amplitude freestream perturbations the strong resonance mechanism associated with fast acoustic waves at the leading edge can trigger a more rapid transition process, compared to the case of slow acoustic waves.

The focus of the present study is on the role of fast and slow acoustic waves in high-amplitude disturbance environments on the receptivity and transition over a 3D generic forebody model. This model was tested in transition experiments conducted by Durant et al. [18] in the Mach 6 Purdue wind tunnel (BAM6QT), both in noisy and quiet conditions. The tests (exploring the natural transition) made in quiet conditions showed the flow remaining laminar over the whole streamwise length, whereas in noisy conditions transition was observed to start just downstream of the nose region, with evidence of streamwise streaks developing off the centerline. Relevant computational studies on the same geometry can be found in the works of Orlik et al. 19] and André et al. 20, 21, which focused on the transition induced by wall injection. 
Three-dimensional (3D) boundary layers in hypersonic flow can include crossflow and attachment-line instabilities. In particular crossflow instability, which is linked to the inflectional crossflow boundary-layer profile forming in regions of local sweep angles and high pressure gradients [22, can dominate in 3D configurations, e.g. on swept wings, axisymmetric cones at an angle of incidence and on rotating disks. Boundary layers in the presence of crossflow instabilities have been studied extensively in low-speed flows, e.g. in the works of Saric et al. 22, Reed and Saric [23], Bippes [24], and have been shown to be characterized, in cases of low freestream disturbances, by the formation of stationary vortices which grow and saturate nonlinearly, providing a pronounced distortion of the mean flow. The breakdown is then caused further downstream by the development of high-frequency secondary instabilities of the deformed mean flow [25, 26].

Crossflow instabilities include both stationary and traveling waves. The stationary modes are in general generated by surface roughness, whereas the traveling modes are linked to vortical freestream disturbances, hence they are expected to be dominant in noisy environments. Traveling crossflow waves are usually found in a relatively low frequency range. Borg et al. 27, for example, detected linearly growing traveling waves in quiet conditions with peak centered at $45 \mathrm{kHz}$, in their Mach 6 experiment over a scaled model of the Hypersonic International Research Experimentation Program's Five (HIFiRE-5) elliptic cone in the Mach 6 Purdue quiet tunnel. However, in contrast to their expectations, they did not observe the presence of traveling waves in noisy conditions. Craig and Saric [28] conducted an experimental investigation of the crossflow instability mechanism in hypersonic flow over a $7^{\circ}$ right circular cone at $5.6^{\circ}$ in the Mach 6 quiet tunnel at Texas A\&M University. Consistent with the low-speed findings, they observed stationary vortices growing and significantly deforming the mean flow, and then attenuating after reaching nonlinear saturation. Moreover, they measured unsteady fluctuations in two different frequency bands, i.e. $15-60 \mathrm{kHz}$ and $80-130$ $\mathrm{kHz}$. The disturbances in the lower frequency band were attributed to growing traveling crossflow waves, in agreement with the results of the nonlinear parabolized stability equations (NPSE) of Kuehl et al. [29] and Oliviero et al. [30, whereas those in the high-frequency band were identified as secondary instabilities. Similar results were obtained experimentally by Ward et al. [31] for a Mach 6 flow over a yawed circular cone.

The works of Choudhari et al. [32, based on linear/nonlinear parabolized stability equations, and of Li et al. 33, based on secondary instability theory, PSE and DNS results, have shed light on the important role played by the growth of the secondary instabilities of primary traveling crossflow waves, as a viable alternate transition scenario when the initial amplitudes of the traveling waves are comparable to those of the stationary modes. Li et al. 34] described, through results from quasiparallel stability theory and PSE, the role of primary traveling crossflow waves (below $100 \mathrm{kHz}$ ) and higher-frequency Mack modes as different sources of secondary instabilities, due to their strong modulation caused by the presence of the stationary

5 of 31

American Institute of Aeronautics and Astronautics 
modes.

Further stability calculations and direct numerical simulations of crossflow instabilities have been carried out for swept wings, circular cones at an angle of attack, and elliptic cones. Balakumar and Owens [35] and Balakumar and King [36] reported substantially higher N-factors for traveling crossflow waves compared to stationary waves in flows over a circular cone at $6^{\circ}$ angle of attack, a swept cylinder and a swept wing with sharp and blunt leading edge. The DNS results of Bartkowicz et al. [37, for the Mach 8 flow over the HIFiRE-5 elliptic cone model, showed the evolution of a system of streamwise vortices forming next to the centerline bulge at different Reynolds numbers, and associated these vortices with stationary crossflow waves. The addition of acoustic noise caused unsteady waves to form on top of the vortices, causing their breakdown at the highest Reynolds numbers. More recent DNS simulations of Dinzl and Candler [38 have shown a difference between the centerline system of vortices, which is a baseflow property linked to the streamlines converging from the attachment line towards the centerline, and the crossflow vortices generated by surface roughness. When distributed roughness was added, a system of crossflow stationary vortices formed in the midspan region between the centerline and the attachment line, whose growth characteristics and wavelength were observed to be very sensitive to the roughness height and Reynolds number, respectively.

Recent studies based on BiGlobal linear stability analysis [39, 40] conducted on the HIFiRE-5 geometry confirmed the dominant role played by the crossflow instabilities in the off-centerline region, but showed also that different instability modes (denoted centerline modes) may occur along the centerline, which can significantly affect transition.

Experimental verification of the important role played by different modes in different flow regions of the HIFiRE-5 geometry, namely the centerline and the off-centerline region, can be found in the work of Juliano et al. [41. Here, surface heat-flux measurements indicated the presence of both centerline and off-centerline transition in noisy conditions. The centerline transition was associated with second-mode waves.

The present work aims to explore through DNS the different transition scenarios associated with fast and slow acoustic waves in a three-dimensional boundary layer, and compare the obtained results with the experimental observations [18, which can provide significant further insight in the transition mechanism due to acoustic noise in hypersonic wind tunnels. Moreover, considering that the acoustic noise radiated from the turbulent boundary layer on the nozzle walls is, in general, known to be the dominant type of disturbances inside hypersonic wind tunnels [42, 43, 44, our study aims also to shed light, through direct comparison between DNS and experimental results, on the relative importance of fast and slow acoustic waves in the environmental noise of hypersonic wind tunnels. For such a purpose, the choice of the imposed disturbance amplitude is crucial to allow reliable comparison between experimental and numerical results. In our simulations, as will be shown later in this paper, the selected amplitude is based on the findings of 
recent experimental as well as numerical studies, e.g. Masutti et al. [45, Parziale et al. [46], Duan et al. [47] and Wagner et al. [48, aimed at investigating the noise characteristics in the environment of hypersonic wind tunnels.

After introducing the numerical method (Section II), the computational mesh (Section III) and the inflow conditions (Section IV), we review the experiments (Section V) in order to size the computational domain (Section VI). The baseflow is presented in Section VII and the main unsteady results in Section VIII.

\section{Numerical method}

\section{II.A. Governing equations}

We consider numerical solutions of the three-dimensional Navier-Stokes equations for compressible flows, written in conservation form, under the assumption of perfect gas. The set of non-dimensional conservation equations in Cartesian coordinates can be written as

$$
\frac{\partial \mathbf{Q}}{\partial t}+\frac{\partial\left(\mathbf{F}_{j}\right)}{\partial x_{j}}=0
$$

In the equation above, $\mathbf{Q}$ is the vector of the conservative variables, while $\mathbf{F}_{j}$ is the vector of the fluxes in Cartesian coordinates. The components of the vectors of the system in conservative form are

$$
\mathbf{Q}=\left[\begin{array}{c}
\rho \\
\rho u \\
\rho v \\
\rho w \\
\rho E
\end{array}\right], \mathbf{F}_{j}=\left[\begin{array}{c}
\rho u_{j} \\
\rho u u_{j}+\delta_{1 j} p-\frac{1}{R e} \tau_{1 j} \\
\rho v u_{j}+\delta_{2 j} p-\frac{1}{R e} \tau_{2 j} \\
\rho w u_{j}+\delta_{3 j} p-\frac{1}{R e} \tau_{3 j} \\
\rho\left(E+\frac{p}{\rho}\right) u_{j}-\frac{1}{R e}\left(u_{i} \tau_{i j}+\frac{\mu}{(\gamma-1) \operatorname{Pr} M^{2}} \frac{\partial T}{\partial x_{j}}\right)
\end{array}\right] .
$$

The terms $\rho, \rho u, \rho v, \rho w$ and $\rho E$ are the conservative variables of the system of equations, where $\rho$ is the density, $u, v$ and $w$ are the velocity components respectively in the $x, y$ and $z$ directions, and $E$ is the total energy per unit mass. The freestream reference values used for the normalization (denoted by the superscript $\left.{ }^{*}\right)$ are $U_{\infty}^{*}, \rho_{\infty}^{*}, \mu_{\infty}^{*}, T_{\infty}^{*}, U_{\infty}^{* 2}, \rho_{\infty}^{*} U_{\infty}^{* 2}$, for velocity, density, viscosity, temperature, energy and pressure respectively. The nose radius $\left(R^{*}\right)$ is chosen as the characteristic length scale, while the characteristic time is given by $R^{*} / U_{\infty}^{*}$. The Reynolds number is defined with respect to the nose radius, as $R e=\left(\rho_{\infty}^{*} U_{\infty}^{*} R^{*}\right) / \mu_{\infty}^{*}$; the Prandtl number is set to 0.72 for air, and $\gamma$ is equal to 1.4 , as we are considering a perfect gas model. The viscous stresses are defined in terms of the velocity derivatives, under the assumption of a Newtonian fluid, as 


$$
\tau_{i j}=\mu\left[\frac{\partial u_{i}}{\partial x_{j}}+\frac{\partial u_{j}}{\partial x_{i}}-\frac{2}{3} \delta_{i j} \frac{\partial u_{k}}{\partial x_{k}}\right]
$$

The dynamic viscosity is obtained through Sutherland's law, with Sutherland's constant equal to 110.4 $\mathrm{K}$ and reference temperature equal to the freestream temperature, $T_{\infty}$. Finally, the system of equations is closed by the nondimensional equation of state for a perfect gas

$$
p=\frac{1}{\gamma M^{2}} \rho T
$$

The system of equations in Cartesian coordinates is transformed into a system of equations in curvilinear coordinates $(\xi, \eta, \zeta)$ as

$$
\frac{\partial \overline{\mathbf{Q}}}{\partial t}+\frac{\partial\left(\overline{\mathbf{F}}_{j}\right)}{\partial \xi_{j}}=0
$$

where the relations between the vectors in curvilinear and Cartesian coordinates are expressed by $\overline{\mathbf{Q}}=J \mathbf{Q}$, $\overline{\mathbf{F}}_{j}=J \mathbf{F}_{i} \frac{\partial \xi_{j}}{\partial x_{i}}$, with $J=\operatorname{det}\|\partial(x, y, z) / \partial(\xi, \eta, \zeta)\|$ being the jacobian of the transformation matrix.

\section{II.B. Modeling of planar oblique acoustic waves}

A three-dimensional (3D) acoustic-wave system, consisting of a main two-dimensional (2D) wave and pairs of opposite-angle lower-amplitude oblique waves, is considered as freestream forcing in the unsteady simulations. The $2 \mathrm{D}$ wave has a wave-vector aligned in the streamwise $(x)$ direction, whereas the oblique waves are considered as planar waves travelling with an angle $\theta$ in the $x z$-plane. The 3D wave model used for the present case is expressed by the following equation, which represents the freestream density fluctuation,

$$
\rho^{\prime}(x, y, t)=\sum_{m=0}^{M_{z}} \sum_{n=1}^{N} A_{m} \cos \left(\beta_{m} z\right) \cos \left(\alpha_{n} x-\omega_{n} t+\psi_{n}\right),
$$

where $\alpha_{n}$ and $\beta_{n}$ are the wavenumbers respectively in the $x$ and $z$ directions, $\omega_{n}$ is the angular frequency, $A_{m}$ is the amplitude of each wave mode, $\psi_{n}$ is a random phase angle, whereas $N$ and $M_{z}$ represent the total number of frequencies and non-zero spanwise wavenumbers respectively. In the present study $N=10$, indicating a spectrum of 10 different frequencies, and $M_{z}=2$, thus giving two spanwise wavenumbers for the oblique waves, $\beta_{1}=2 \pi m / L_{z}$ (with $L_{z}$ being the length of the domain in the $z$ direction). Note that for $m=0$ we have $\beta_{0}=0$, which is a $2 \mathrm{D}$ wave. The streamwise wavenumber, $\alpha_{n}$, is linked to the angular frequency, $\omega_{n}=2 \pi f_{n}$, through the relation $\alpha_{n}=\omega_{n} /(1 \pm 1 / M)$, in which the term $1 \pm 1 / M$ represents the freestream phase speed in the $x$ direction of the acoustic waves, with the plus sign indicating fast acoustic waves, and the minus sign standing for slow acoustic waves. For each frequency, and for each spanwise 
wavenumber $m=1,2$, two waves with the same amplitude $A_{m}$ and opposite angle $\theta_{m, n}= \pm \arctan \left(\frac{\beta_{m}}{\alpha_{n}}\right)$ are inserted, such that the $z$-velocity perturbation relative to each wave $\left(w^{\prime}= \pm(1 / M) \rho^{\prime} \sin \theta\right)$ cancels. This gives $w^{\prime}=0$ identically in the freestream. Finally, the relation between the amplitude of each single oblique wave and that of the main $2 \mathrm{D}$ wave is $A_{m}=\frac{1}{4} A_{0}$ (for $m=1,2$ ) at each frequency, and the amplitude $A_{0}$ is independent of frequency.

\section{Geometry and mesh}

We consider now the three-dimensional geometry of a generic forebody model, proposed by MBDAFrance, for which transition experiments were performed by Durant et al. [18] in the Mach 6 hypersonic wind tunnel of the Purdue University. Figure 1 shows different views of the geometry. It consists of a wedge with a rounded leading-edge shape, nose radius of $1.25 \mathrm{~mm}$ (in the $x y$-plane), a length of $340 \mathrm{~mm}$, maximum width along the $z$-axis of $120 \mathrm{~mm}$, maximum height along the $y$-axis of $50 \mathrm{~mm}$, and half-wedge angle (in the $x y$-plane, with reference to the side view in 1 of $4^{\circ}$. In our numerical simulations we consider a case with zero angle of attack and zero yaw angle, namely the flow direction is aligned with the $x$-axis of the body, and symmetry with respect to both the $x y$ and $x z$ planes (considering the origin of the reference system on the tip of the body) is assumed, including the upstream disturbances.

The grid is a body-fitted structured single-block grid. In each $x y$ plane, the j-lines (the wall-tangential grid lines) are gradually adapted from the wall shape to the shock curve projected onto this plane, whereas the i-lines are normal to the wall. In an $x z$ plane, the k-lines are initially aligned with the leading-edge shape, then they gradually become perpendicular to the centerline, as shown in figure 2 , A stretching is applied along the wall-normal direction, in order to increase the resolution towards the wall and at the shock. Stretching is also performed in the $x$ direction in order to increase the resolution in the leading-edge region. A detailed description of the methodology used to generated the shock-adapted grid, as well as the stretching function applied, can be found in Cerminara [49]. Figure 2 shows the mesh details, in the $x z$-plane, of the surface in the leading-edge region. As can be seen, the point distribution is denser near the leading-edge, in order to resolve the high gradients characterising this zone of the flowfield, and gets coarser downstream. The internal grid lines are adjusted to follow the shape of the three-dimensional shock. 


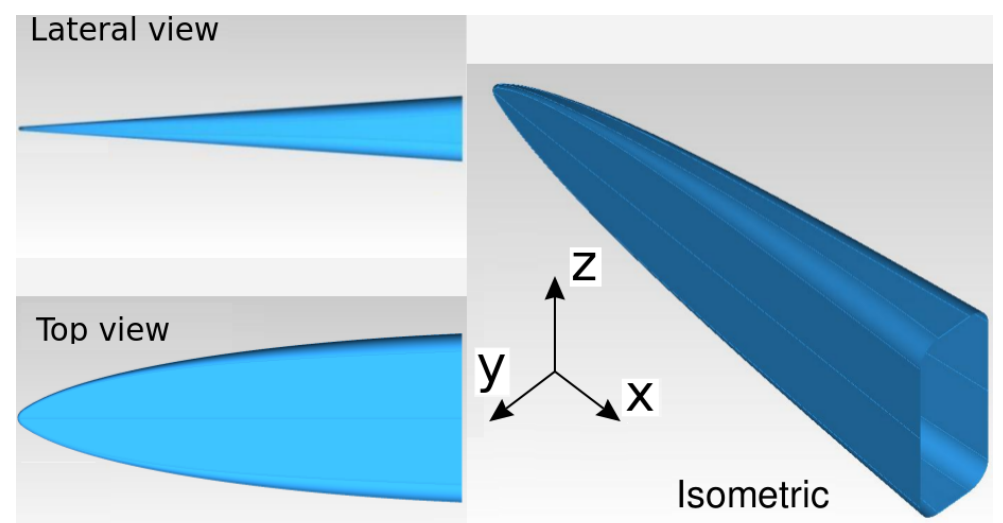

Figure 1: Lateral, top (on the $x z$-plane) and isometric views of the MBDA forebody geometry

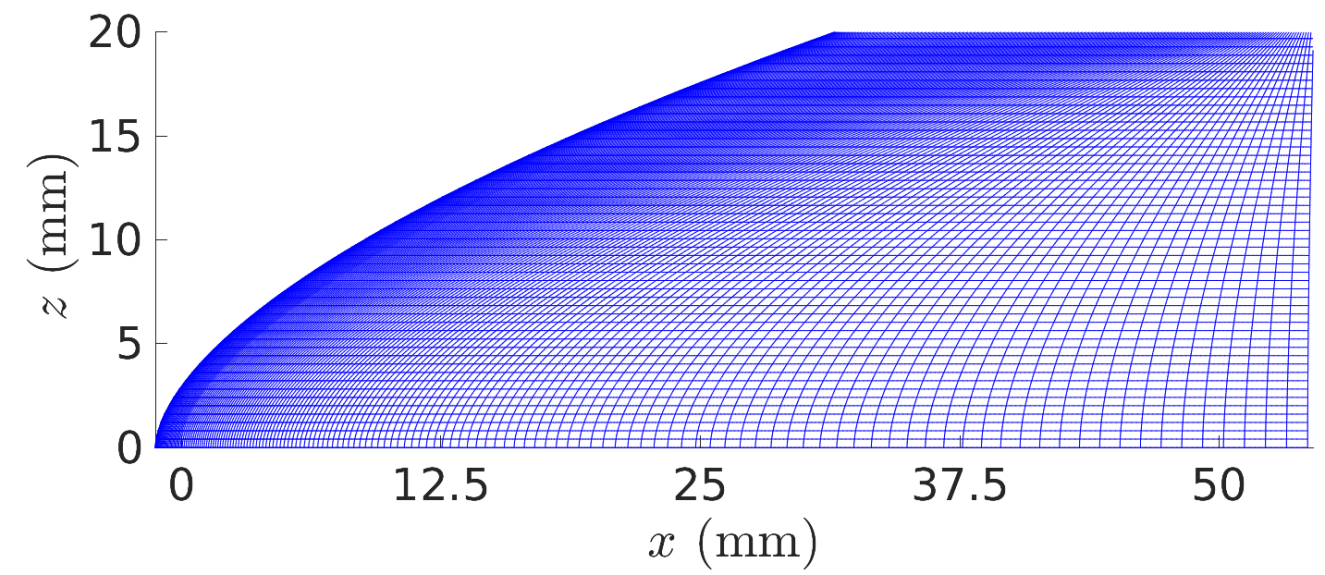

Figure 2: Example of a coarse mesh in the leading-edge region of the computational domain. Top view of the surface

To simplify the mesh generation, the side boundary of the computational domain is obtained by cutting the geometry along the longitudinal direction at a particular spanwise distance from the symmetry axis. The side boundary condition consists of a zero-gradient condition, which sets the gradients of each quantity along the grid lines to zero, namely $\frac{\partial()}{\partial \tau}=0$, with $\tau$ indicating the direction tangential to the $k$-grid lines. The use of the zero-gradient boundary condition is motivated by an observation that at sufficiently high distances from the centerline $(z=0)$ the gradients of the physical quantities in the tangential direction are small. Figure 3 shows the surface density field for a body with a width of $40 \mathrm{~mm}$. As can be seen, the high gradients are confined in the near-centerline region, while the off-centerline region is characterised by much smaller gradients.

The above-described approach to obtain the computational domain presents the following advantages: i) it provides a uniform spanwise resolution, so that the breakdown region downstream is treated with the same spanwise resolution of the upstream receptivity region; and ii) it limits the number of points in the leading-edge region, avoiding a too-small $z$-spacing downstream, which would be a main issue for a grid built 
through grid lines in the radial (normal to the leading edge) direction.

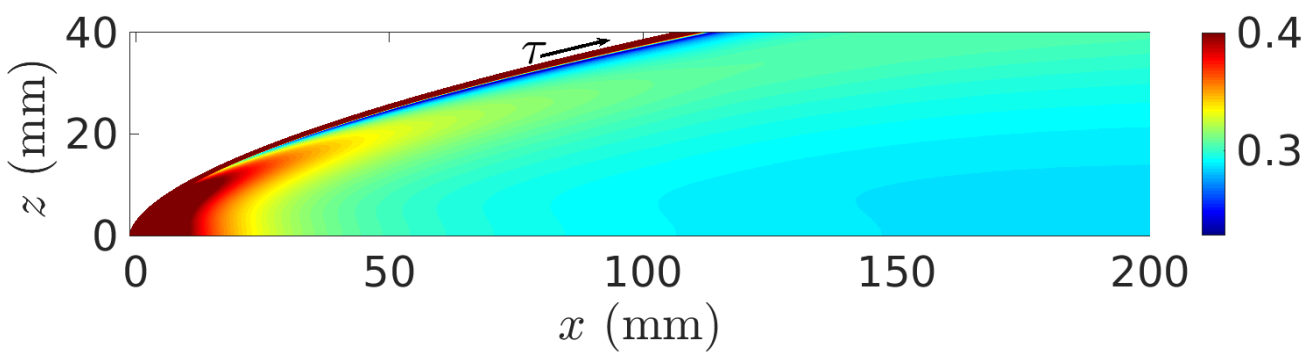

Figure 3: Density field on the wall surface

\section{Flow conditions and settings of the numerical simulations}

The flow conditions of the present numerical case reproduce the freestream of the Mach 6 low-enthalpy wind tunnel at Purdue University. The experiments were carried out for different values of the unit Reynolds number $\left(R e_{m}\right)$, ranging from $2.3 \times 10^{6}$ to $13 \times 10^{6} / \mathrm{m}$. In our numerical study a unit Reynolds number of $4.6 \times 10^{6} / \mathrm{m}$ is considered, corresponding to one of the transitional cases in the experiments. The other flow conditions are $M=6, T_{\infty}^{*}=51.7 \mathrm{~K}, T_{0}^{*}=424 \mathrm{~K}, p_{0}^{*}=4.16 \times 10^{5} \mathrm{~Pa}, T_{w}^{*} / T_{\infty}^{*}=5.8$. The latter condition for the wall temperature ratio corresponds to an isothermal wall with temperature assumed equal to $300 \mathrm{~K}$.

On the $x y$ and $x z$ symmetric planes a symmetric boundary condition is adopted. A fixed uniform inflow boundary condition is set on the inlet boundary, and a zero-gradient boundary condition is used on both the side and the outlet boundaries. When unsteady simulations are performed, the 3D acoustic-wave model described in Section II.B is used as inflow on the inlet boundary. The disturbance field is assumed to be symmetric with respect to both the $x y$ and $x z$ symmetry planes of the body.

The code used to carry out the numerical simulations is known as SBLI (Shock-Boundary-Layer-Interaction), developed over a number of years at the University of Southampton, consisting of a $4^{\text {th }}$-order central differencing scheme, as base scheme, in conjunction with a $2^{n d}$-order Harten-Yee TVD (Total-Variation-Diminishing) shock-capturing scheme [50, as a filter step. Validation of the code can be found in the works of Sandham et al. [51] and De Tullio et al.552.

\section{Natural transition experiments}

Figure 4 shows the probe, made of aluminum, used in the transition experiments of Durant et al. [18. Wall heat-flux measurements performed by Temperature-Sensitive Paint (TSP) were used to evaluate boundary-layer transition on the body surface, and two Schmidt-Boelter gauges were used to calibrate the TSP during post-processing. The luminophore used in the TSP was Ru(bpy). It was applied after a base coat of white primer and spray paint, which increases the signal-to-noise ratio. In addition, 15 flush-mounted 
pressure sensors (10 PCB132A31 and 5 Kulite XCQ-062-15A) were inserted for pressure measurements. Figure 5 shows the results for the wall heat flux $\left(\mathrm{kW} / \mathrm{m}^{2}\right)$ at two Reynolds numbers for noisy wind-tunnel conditions. As can be seen, at the lowest unit Reynolds number $\left(R e_{m}=2.0 \times 10^{6} / \mathrm{m}\right)$ the low values of the heat flux indicate that the flow was laminar over the whole surface of the wedge, while at $R e_{m}=4.6 \times 10^{6} / \mathrm{m}$ transition is observed to take place at approximately a distance of $150 \mathrm{~mm}$ from the nose tip (note that the nose tip is not included in the figure, and its position in the figure reference system is about $x=-100 \mathrm{~mm}$ ).

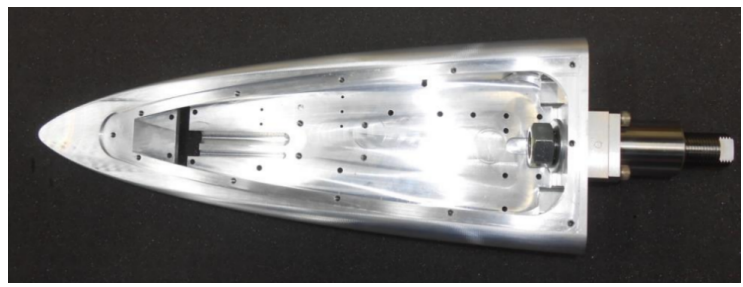

Figure 4: Lower surface of the probe, cover part removed

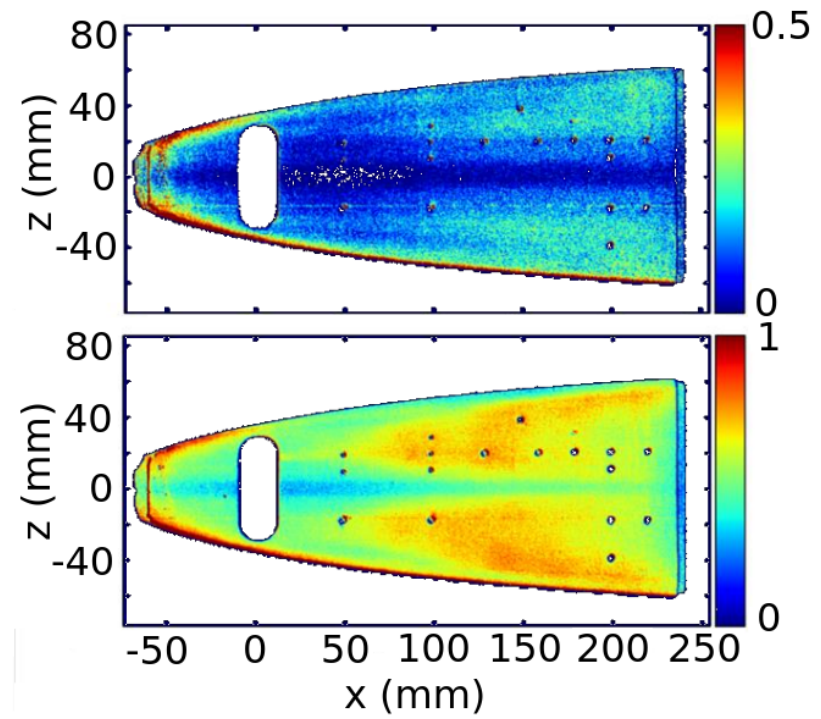

Figure 5: Wall heat-flux $\left(\mathrm{kW} / \mathrm{m}^{2}\right)$ at $R e_{m}=2.0 \times 10^{6} / \mathrm{m}$ (top) and $R e_{m}=4.6 \times 10^{6} / \mathrm{m}$ (bottom). Same $x$-axis for both figures

\section{Experimental observations and domain sizing}

The observation of the experimental results [18, at the target unit Reynolds number considered in the numerical simulations $\left(R e_{m}=4.6 \times 10^{6} / \mathrm{m}\right)$, allows us to set the minimum required size of the computational domain to solve the most relevant flow regions for the receptivity-breakdown mechanism and to capture the main transition patterns obtained in the experiments. In figure 6 the dimensional wall heat flux $\left(\mathrm{kW} / \mathrm{m}^{2}\right)$ obtained in the experiment at $R e_{m}=4.6 \times 10^{6} / \mathrm{m}$ is shown. This experimental case was performed under noisy wind tunnel conditions. As can be seen, the highest heat-flux values are reached in the front leadingedge region, as expected from the high temperatures reached in the shock layer near the nose. In the 
downstream region, the wall heat flux assumes low values at the centerline, thus suggesting that the boundary layer near the symmetry plane is laminar; however, the off-centerline region is characterised by high heat flux values, starting from about $x=50 \mathrm{~mm}$, which suggests, in contrast, that the boundary layer here undergoes transition to turbulence.

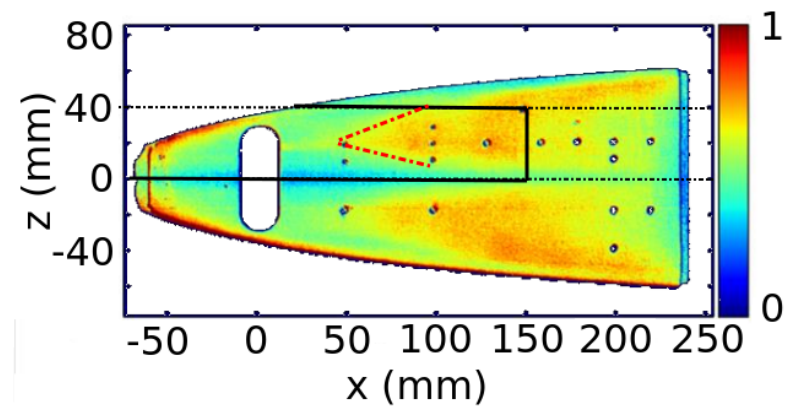

Figure 6: Edges of the computational domains drawn on the wall heat-flux result $\left(\mathrm{kW} / \mathrm{m}^{2}\right)$

From the wall heat-flux experimental results the following observations can be made: i) transition appears to start at a streamwise distance from the tip between about $140 \mathrm{~mm}$ and $150 \mathrm{~mm}$, and at a distance approximately of $20 \mathrm{~mm}$ from the centerline; ii) the transition front spreads downstream forming a wedge, as indicated by the red dashed lines drawn along the edges of the early transition front; iii) thus, transition might be induced by disturbances coming from the early leading-edge region and developing downstream at a distance from the centerline of approximately one-third of the maximum half-body width $\left(L_{z}^{*} / 2=60\right.$ $\mathrm{mm})$.

These considerations allow us to shape the computational domain to investigate the main transition mechanism. The black solid lines drawn in figure 6 represent the edges of the computational domain. The domain is $40 \mathrm{~mm}$ wide in the $z$ direction (namely $L_{z}=32$ in nondimensional coordinates) and $250 \mathrm{~mm}$ long in the $x$ direction. The grid size used to carry out our numerical simulations is $2120 \times 300 \times 400\left(N_{x} \times N_{y} \times N_{z}\right)$, which was observed to provide a grid-independent solution. A sensitivity analysis of the laminar baseflow solution to the domain size in the spanwise direction and to the side boundary condition can be found in the work of Cerminara et al. [53]. Results obtained for two different domain widths, namely $30 \mathrm{~mm}$ and $40 \mathrm{~mm}$, with grid sizes of $2120 \times 300 \times 300$ and $2120 \times 300 \times 400$ respectively, indicated that the internal solution is not sensitive to the domain width. A slight effect from the side boundary was observed to be confined within a narrow region adjacent to the side boundary. Streamlines within the boundary layer were also presented in [53, which indicated flow converging from the attachment line towards the centerline, due to the crossflow effect. The influence of the streamlines coming from outside the side boundary (which are not considered in our simulations) was found to be confined in a limited region adjacent to the side boundary, downstream of the transition point observed in the experiment. Thus, it was concluded that our computational domain, with the considered size, is able to capture the main receptivity mechanism in the leading-edge region, as 
well as the streamwise evolution of the induced disturbances up to the observed transition location.

\section{Laminar base flow results}

The leading edge is a critical zone, determining the general flowfield characteristics. This is due to the crossflow that forms inside the boundary layer, due to the imposed pressure gradients. The wedge junction is defined as the interface point between the circle and the wedge profile of the wall in a $x y$ plane, indicated by an arrow in figure 8. As the flow coming from the leading edge approaches this point, a small region of strong crossflow (represented by a thin dark blue zone) forms inside the boundary layer. We refer to this region around the junction point as the wedge-junction region. The near-wall flow entering the wedge with an inwards (i.e. towards the centerline) orientation generates inflectional crossflow boundary-layer profiles in the zone of the wedge junction, as can be observed in figure 7 which shows crossflow profiles at several $x$ positions. Figure 8 for the contours of the crossflow velocity $(w)$ on the $x y$-plane at $z=30 \mathrm{~mm}$, shows that the wedge-junction region is characterized by highest negative peak (represented by the blue region) of the crossflow.

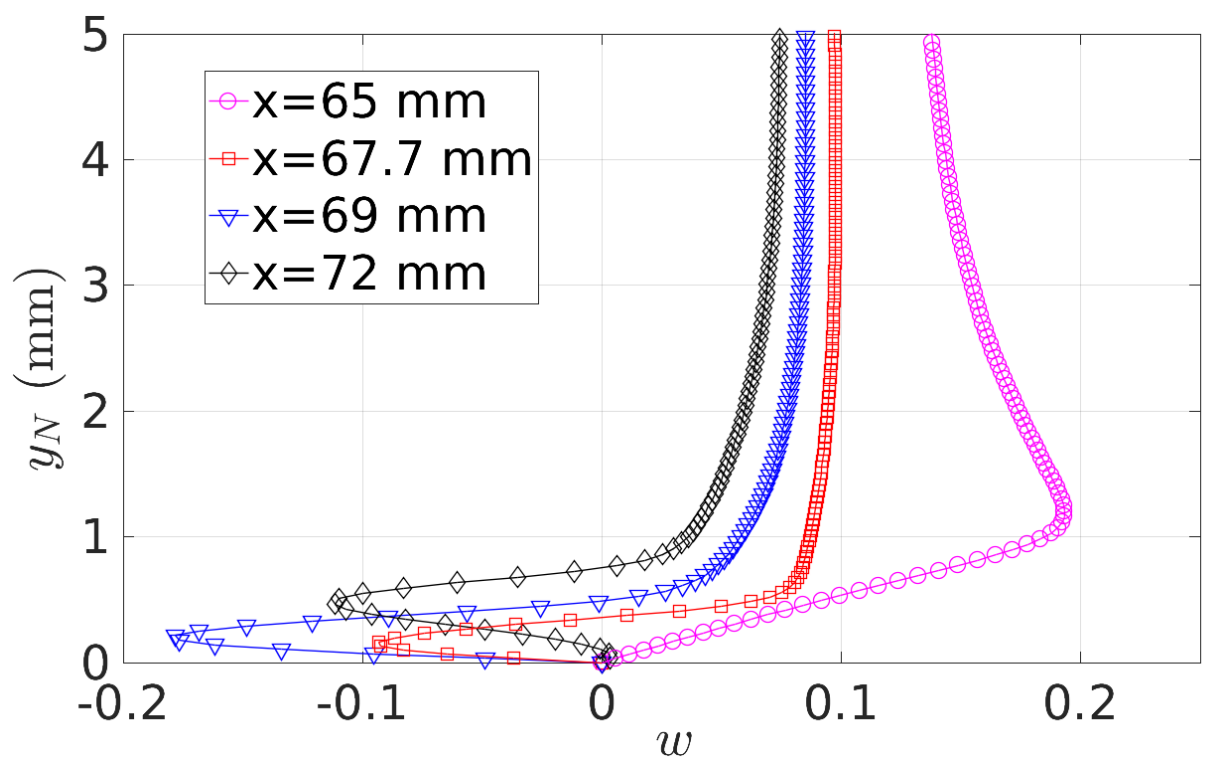

Figure 7: Crossflow boundary-layer profiles at different streamwise locations and at $z=30 \mathrm{~mm}$ 


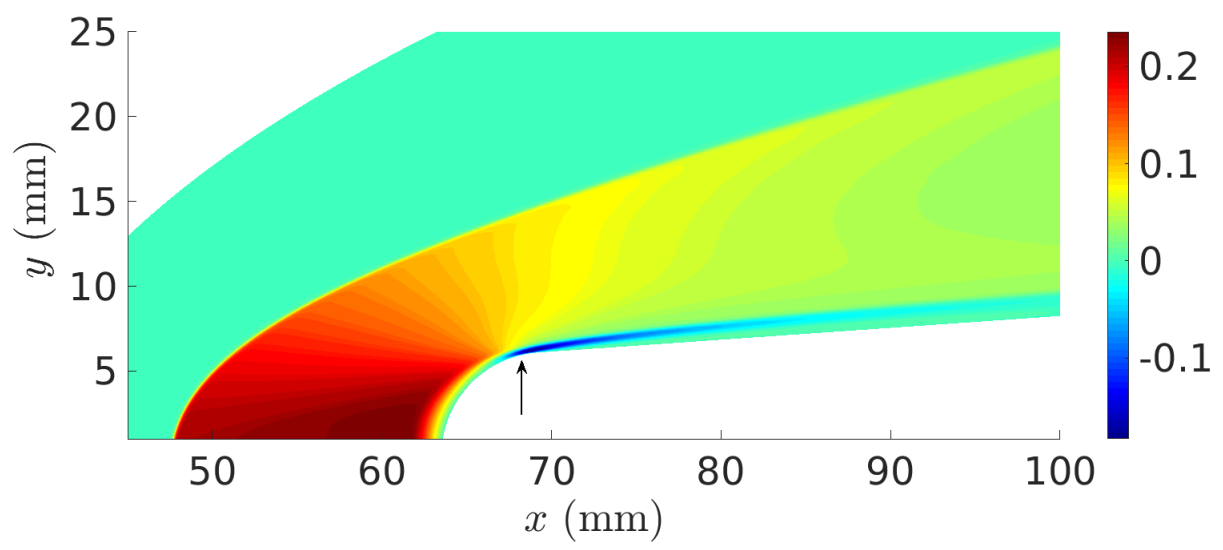

Figure 8: Crossflow $(w)$ contours in the $x y$-plane at $z=30 \mathrm{~mm}$

The inflectional crossflow profiles have, as is known, an important impact on the boundary-layer stability. However, there is another important effect associated with the crossflow at the wedge junction, which has a direct impact on the local wall heat flux. Namely, the boundary layer becomes very thin at the wedge junction, due to the 3D effects related to the crossflow, hence, as a result, the outer cold fluid gets very close to the wall and produces a reverse of the wall heat flux, such that the fluid is heated by the wall in this region. This can be seen in figure 9, showing the temperature boundary-layer profiles as a function of the wall-normal coordinate $y_{N}$ at the same points of the crossflow profiles plotted in figure 7. At $x=65 \mathrm{~mm}$, which is ahead of the wedge junction, the maximum temperature is reached inside the boundary layer, but as soon as the wedge junction is reached and the negative crossflow develops, the boundary-layer thickness decreases dramatically and the temperature gradient at the wall becomes negative.

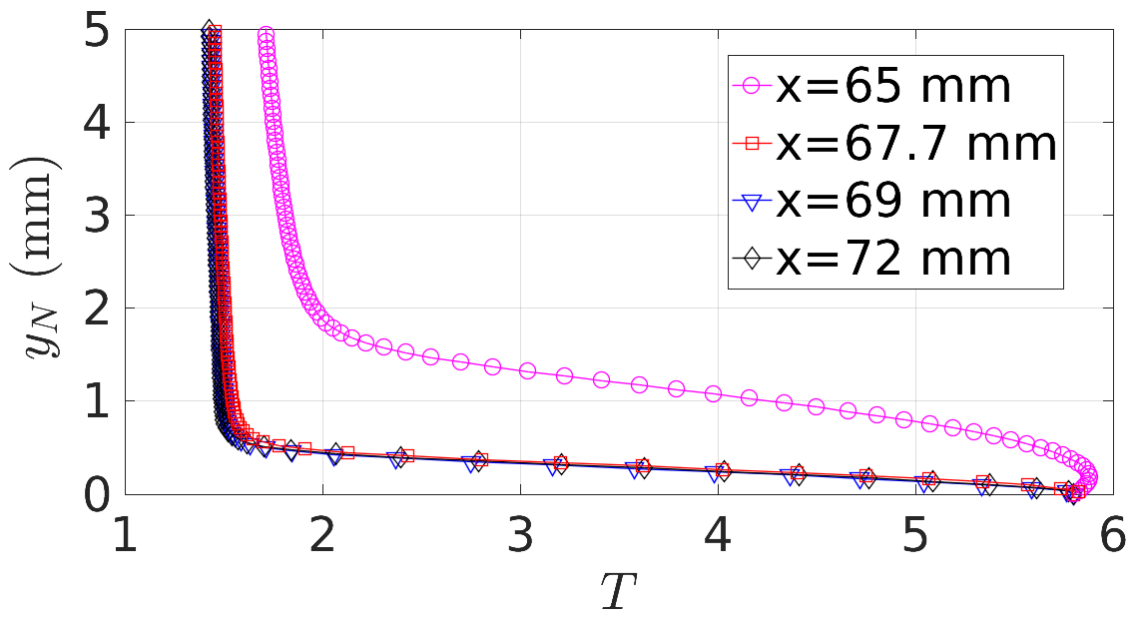

Figure 9: Temperature $(T)$ boundary-layer profiles at different streamwise locations and at $z=30 \mathrm{~mm}$

In figure 10, which shows a close-up of the wall heat flux at the leading edge, a narrow region of high negative (blue) heat-flux values, corresponding to the wedge-junction zone, is observed. The reversal of the surface heat flux at the wedge junction can have important implications on the transition process and on the 
thermal-protection performance of a hypersonic vehicle. For example, in the presence of chemical surface reactions (e.g. oxidation), a high spatial gradient of the heat flux might produce localised gradients in the production/consumption rate of the reaction, resulting in gradients of the mass-loss rate and of the surface regression. Abrupt changes of the surface geometry might, in turn, induce transition through roughness.

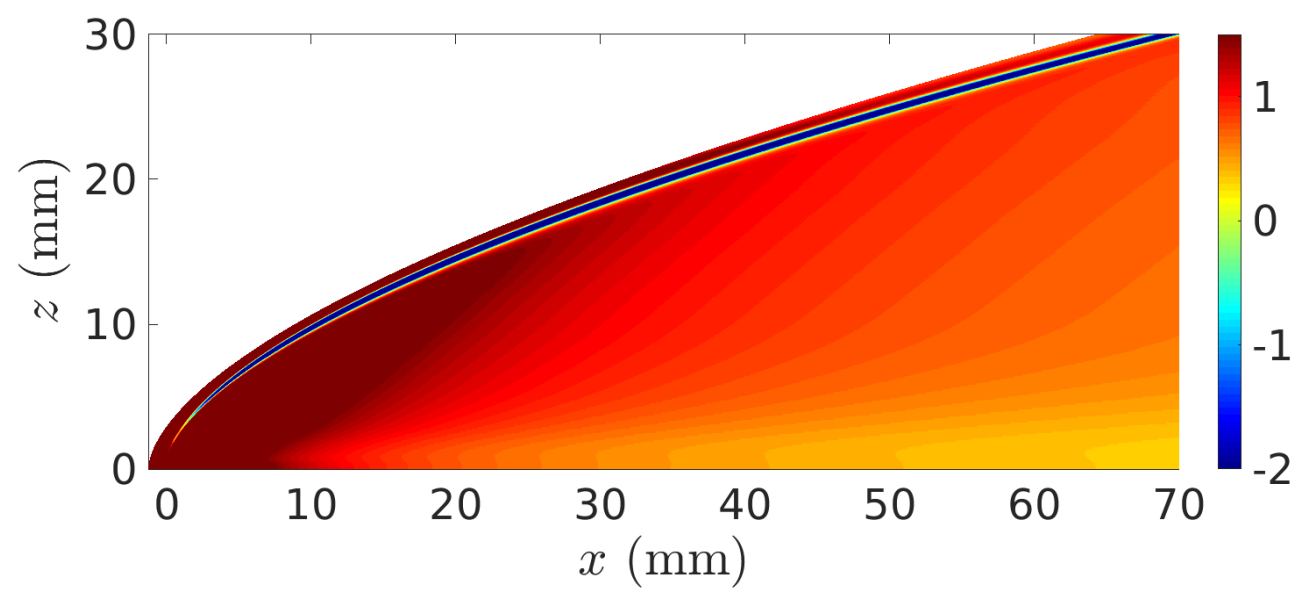

Figure 10: Wall heat flux $\left(\mathrm{kW} / \mathrm{m}^{2}\right)$ in the leading-edge region. Negative values located at the wedge junction

Figures 11 and 12 show temperature and crossflow velocity $(w)$ cross sections (in the $z y_{N}$-plane) respectively, at the streamwise position $x=40 \mathrm{~mm}$. The right edge of the plots corresponds to the spanwise position of the wedge junction. The figures highlight how the boundary layer gets rapidly thinner along the spanwise direction towards the wedge junction. The result in figure 12 shows the high inwards crossflow velocity magnitudes reached at the wedge junction, compared to the other spanwise positions on the wedge. This indicates that a stream coming from the leading edge and flowing towards the centerline is released by the wedge junction into the flowfield over the wedge surface.

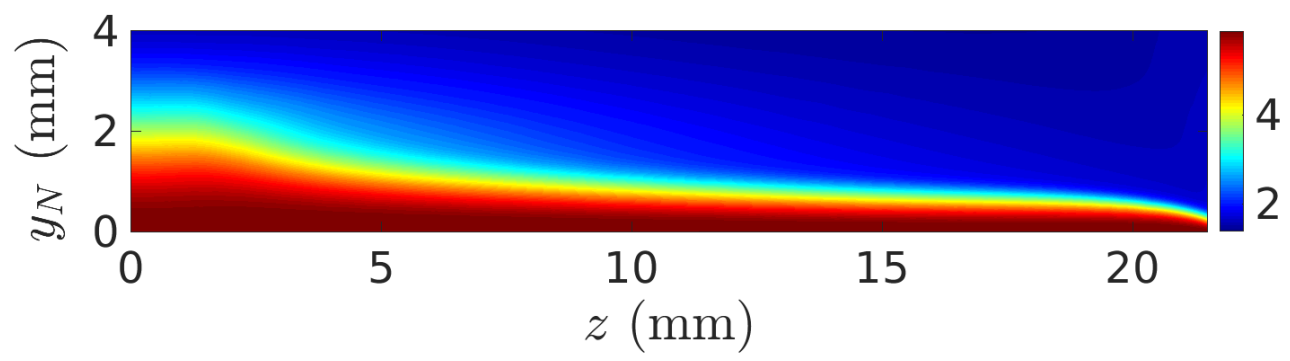

Figure 11: Temperature $(T)$ contours in the $z y_{N}$-plane at the streamwise position $x=40 \mathrm{~mm}$ 


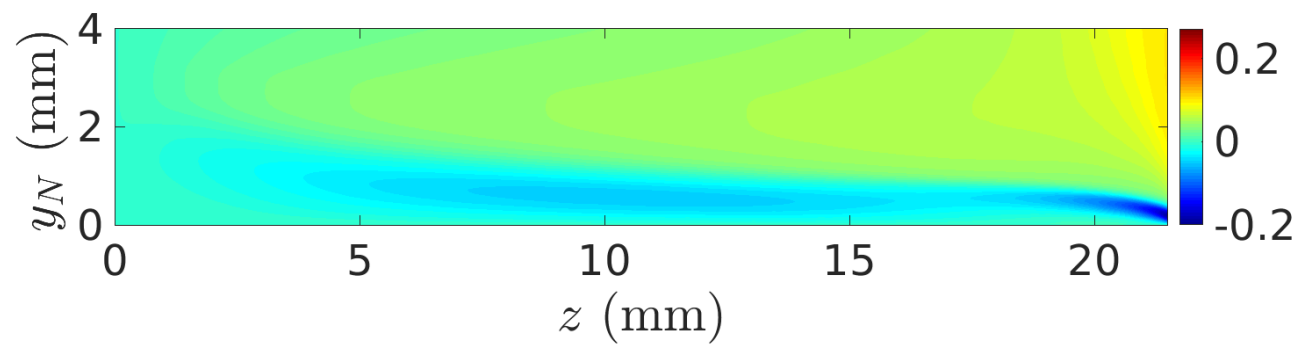

Figure 12: Crossflow $(w)$ contours in the $z y_{N}$-plane at the streamwise position $x=40 \mathrm{~mm}$

\section{Results for the unsteady simulations}

\section{VIII.A. Transition patterns with fast and slow acoustic waves}

We now present numerical results for the unsteady simulations with three-dimensional freestream fast and slow acoustic waves (the wave model was described in Section [I.B. , with the aim of understanding the transition mechanism associated with either type of waves. The numerical results will then be compared with the experimental results.

In order to reduce eventual disturbance reflection effects from the side boundary into the internal flowfield, a $z$-dependent hyperbolic-tangent-based damping function, $h(z)$, was applied over the 3D wave model in Section II.B which gradually damps out the amplitude of all the imposed freestream disturbance waves (both 2D and oblique) along the $z$-direction from a certain position $\left(z_{0}\right)$ up to the side boundary. Hence, the modified 3D wave model can be expressed as,

$$
\begin{gathered}
\rho^{\prime}(x, z, t)=\sum_{m=0}^{M_{z}} \sum_{n=1}^{N} A_{m} h(z) \cos \left(\beta_{m} z\right) \cos \left(\alpha_{n} x-\omega_{n} t+\psi_{n}\right), \\
h(z)=1-\frac{1}{2}\left(\tanh \left(\frac{S\left(z-z_{0}\right)}{L_{z}^{*}-z_{0}}\right)+1\right),
\end{gathered}
$$

in which $S=10$, and $z_{0}=26.5 \mathrm{~mm}$. It should be noted that, while a random phase angle, $\psi_{n}$, is imposed in the streamwise direction, the phase angle in the spanwise direction is fixed to zero so that to guarantee the symmetry of the freestream disturbance field with respect to the $z=0$ symmetry plane. For each single frequency, the freestream amplitude of the $2 \mathrm{D}$ wave (based on the density) is set to $A_{0}=2.5 \times 10^{-2}$, whereas each oblique wave is set with an amplitude of $1 / 4$ that of the main $2 \mathrm{D}$ wave. The overall amplitude of the wave system (the sum between the $2 \mathrm{D}$ and the oblique waves) is $A_{\infty}=5 \times 10^{-2}$. The level of 2.5 $\%$ for the 2D wave is of the same order as that measured by Masutti et al. [45] (4.9\%) for the density fluctuations in the Mach 6 blowdown facility at VKI, while the level relative to the overall wave system (5 
\%) reproduces this reference measured value very well. The 3D modes are set with an amplitude (0.625\%) similar to the levels reported by Parziale et al. [46, who measured density levels of $0.5 \%$ for the Mach 5.5 flow in a T5 reflected-shock tunnel, Duan et al. 47, who computed a value of $0.39 \%$ for the pressure fluctuations radiated by a turbulent boundary layer in a Mach 2.5 flow, and Wagner et al. [48, who estimated a similar value of $0.48 \%$ for the noise in a Mach 3 flow in the case of dominant slow acoustic waves, using a joint experimental-numerical approach. These disturbance levels were chosen in order to simulate noisy wind-tunnel conditions, thus allowing for nonlinear mechanisms to be triggered in the boundary layer. A set of 10 separate frequencies were introduced, ranging from $f_{1}=0.0105$ to $f_{10}=0.105$, corresponding to the dimensional values of $f_{1}^{*}=7.3 \mathrm{kHz}$ and $f_{10}^{*}=73 \mathrm{kHz}$. The considered frequency range captures the frequency band in which traveling crossflow waves were detected in several experimental and computational works [28, 31, 34, as described in Section I. Moreover, in the work of Wagner et al. [48] low frequencies (below $100 \mathrm{kHz}$ ) were found to have a significant effect on the response to slow acoustic waves, which was more amplified compared to the higher frequencies.

Figures 13 and 14 show the time-averaged surface heat flux (in $\mathrm{kW} / \mathrm{m}^{2}$ ) for fast and slow acoustic waves respectively. As can be seen, the surface heat-flux structure in the case of fast acoustic waves show the presence of wavefronts nearly perpendicular to the flow direction, which is consistent with the 2D fast mode being the dominant mode in the nose region, as shown in [16], due to the powerful resonance mechanism, and leading to a modulated response further downstream. The case of slow acoustic waves (figure 14) shows a fundamentally different structure, that is characterised by the presence of elongated streamwise-oriented streaks generated in the early leading-edge region. In particular, three streaky structures can be distinguished (whose position and direction is indicated by arrows in figure 14). One forms close to the centerline, near $z=10 \mathrm{~mm}$, between $x=50 \mathrm{~mm}$ and $x=100 \mathrm{~mm}$, and then decays rapidly downstream. Another, with higher spanwise size, is located near the side boundary at about $z=35 \mathrm{~mm}$, forming just downstream of the leading edge and gradually decaying downstream. A third streak originates in the leading-edge zone at about $z=20 \mathrm{~mm}$, which grows until it undergoes breakdown after $x=200 \mathrm{~mm}$.

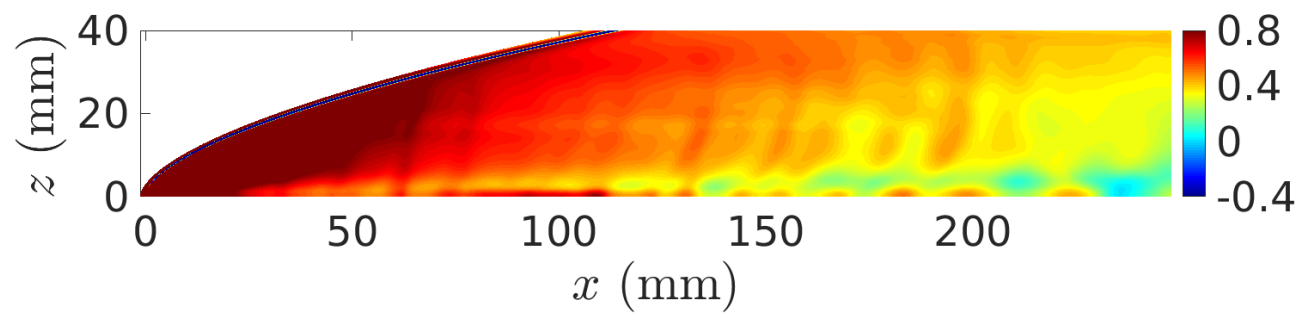

Figure 13: Time-averaged surface heat flux $\left(\mathrm{kW} / \mathrm{m}^{2}\right)$ for fast acoustic waves 


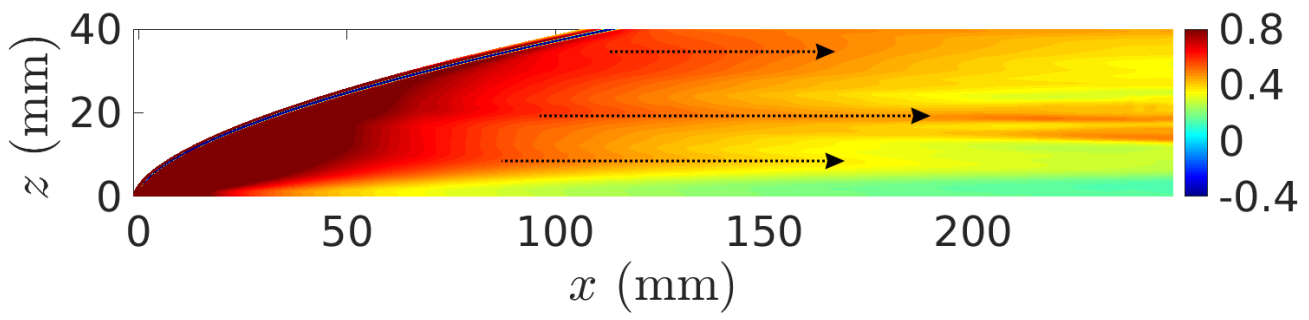

Figure 14: Time-averaged surface heat flux $\left(\mathrm{kW} / \mathrm{m}^{2}\right)$ for slow acoustic waves. The arrows indicate the streamwise streaks

Another noticeable difference between the fast-wave and slow-wave results is observed for the solution in the near-centerline region, which suggests that in the slow-wave case the boundary layer along the symmetry plane is laminar through the whole domain length, whereas, in the fast-wave case, a highly perturbed boundary layer is obtained, with a nonlinear growth in the region $x=70 \mathrm{~mm}-150 \mathrm{~mm}$. This is due to the amplification of the $2 \mathrm{D}$ fast mode in the upstream nose region in the fast-wave case. The centerline is indeed the region where the flow is most sensible to the $2 \mathrm{D}$ incident modes, as the baseflow here is quasi two-dimensional, due to the symmetry condition and the small local leading-edge sweep angles.

In figure 15 a close-up of the heat flux for the slow-wave case in the downstream region is shown, providing more details of the nonlinear growth of the streamwise streak located at $z=20 \mathrm{~mm}$. A secondary streak adjacent to the main one is formed at about $x=200 \mathrm{~mm}$, which rapidly grows in amplitude and starts spreading downstream, deflecting its path towards the centerline.

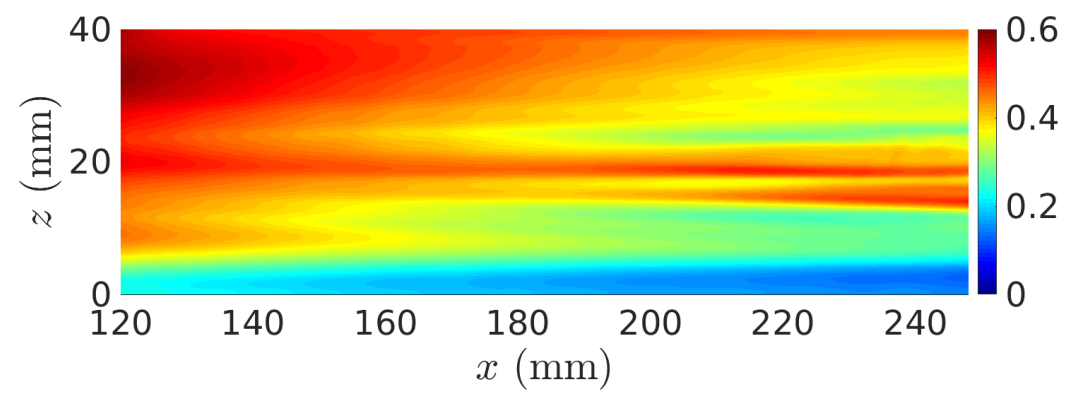

Figure 15: Time-averaged surface heat flux $\left(\mathrm{kW} / \mathrm{m}^{2}\right)$ for slow acoustic waves. Close-up in the downstream region

The results for the surface heat flux indicate that the solution obtained with slow acoustic waves is closer to the experimental results observed in figure 6, which showed an off-centerline transition core and a laminar boundary layer near the centerline. A direct comparison between numerical and experimental results for the slow-wave solution is shown in figure 16, where the experimental figure has been adapted to match the scale of the numerical plot. 


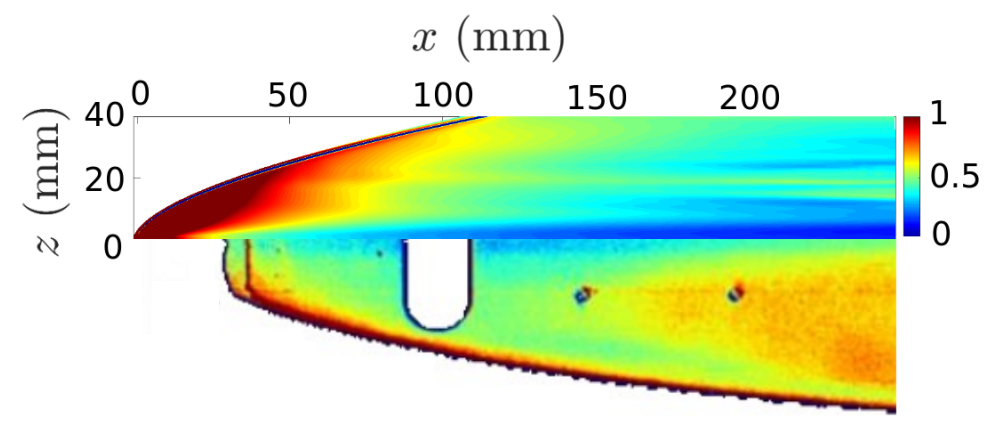

Figure 16: Comparison between numerical (top half) and the experimental (bottom half) surface heat flux $\left(\mathrm{kW} / \mathrm{m}^{2}\right)$

The comparison shows similar heat-flux levels reached along the leading edge and in the upstream region of the wedge, which decrease towards the centerline, where the blue values indicate a laminar boundary layer. However, the high (red) heat-flux zone near the leading edge appears to cover a larger area in the numerical result, which might be due to calibration effects of the experimental apparatus used to compute the heat flux, as well as to differences in amplitude between the applied 3D wave model and the real noise in the experiments. The main difference between the numerical prediction and the experimental results is observed downstream of $x=150 \mathrm{~mm}$, where, in the experimental case, a wedge-shaped transition front is seen to develop and spread symmetrically downstream, affecting also the near-centerline region at longer distances. In contrast, in the numerical case, the heat flux near the symmetry plane keeps decreasing through all the domain length and no transition front is observed until the distance of about $x=230 \mathrm{~mm}$ is reached, where the streamwise streak located at $z=20 \mathrm{~mm}$ begins the nonlinear breakdown process.

Hence, the experimental results show a more rapid transition process, which may suggest the presence of a higher noise level in the wind tunnel, compared to the freestream disturbance amplitude used in the numerical simulations. In addition, the pressure transducer placed upstream of the position $x=150 \mathrm{~mm}$, and at approximately $z=20 \mathrm{~mm}$, as figure 16 clearly shows, might have worked as a roughness element during the experimental tests, thus enhancing the transition process downstream. In order to see more details of the breakdown process taking place in the numerical solution, we show in figure 17 the instantaneous contours of the streamwise wall-tangential velocity component $\left(u_{t}\right)$ in the $x y$-plane along the $j=15$ grid lines inside the boundary layer.

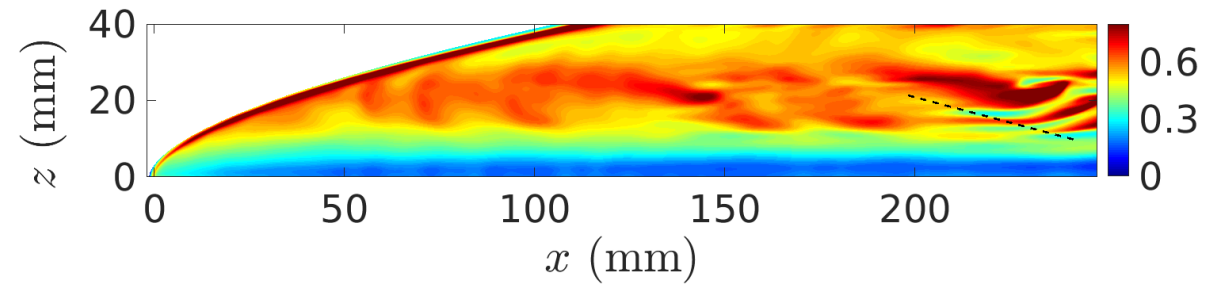

Figure 17: Wall-tangential velocity $\left(u_{t}\right)$ on the $j=15$ grid lines inside the boundary layer, for slow waves. 
Here the main disturbances coming from the leading edge at about $z=20 \mathrm{~mm}$, within the crossflow dominated region, are seen to undertake a nonlinear growth just upstream of $x=150 \mathrm{~mm}$, which induces a transition process further downstream. This transition process, in turn, is observed to spread downstream forming a series of strong velocity spikes propagating towards the centerline with an inclination angle (indicated by the dashed line in the figure) close to the wedge-shaped transition front angle shown by the experimental results. Hence, the numerical solution inside the boundary layer shows transition patterns and location more similar to those observed in the experimental results at the wall. The numerical solution indicates that the transition process starts away from the wall, and that the perturbations related to the nonlinear breakdown process have not yet reached the wall.

\section{VIII.B. Slow-mode receptivity and streak evolution}

As shown in Section VIII.A, the numerical solution with slow acoustic waves is closer to the experimental results in terms of transition patterns, so, in this Section, we focus on the results for the slow-wave case. The receptivity process is studied through contours of the Fourier transformed wall pressure fluctuations (real part) at different frequencies, $f_{3}, f_{4}, f_{6}$ and $f_{8}$, shown in figure 18 .

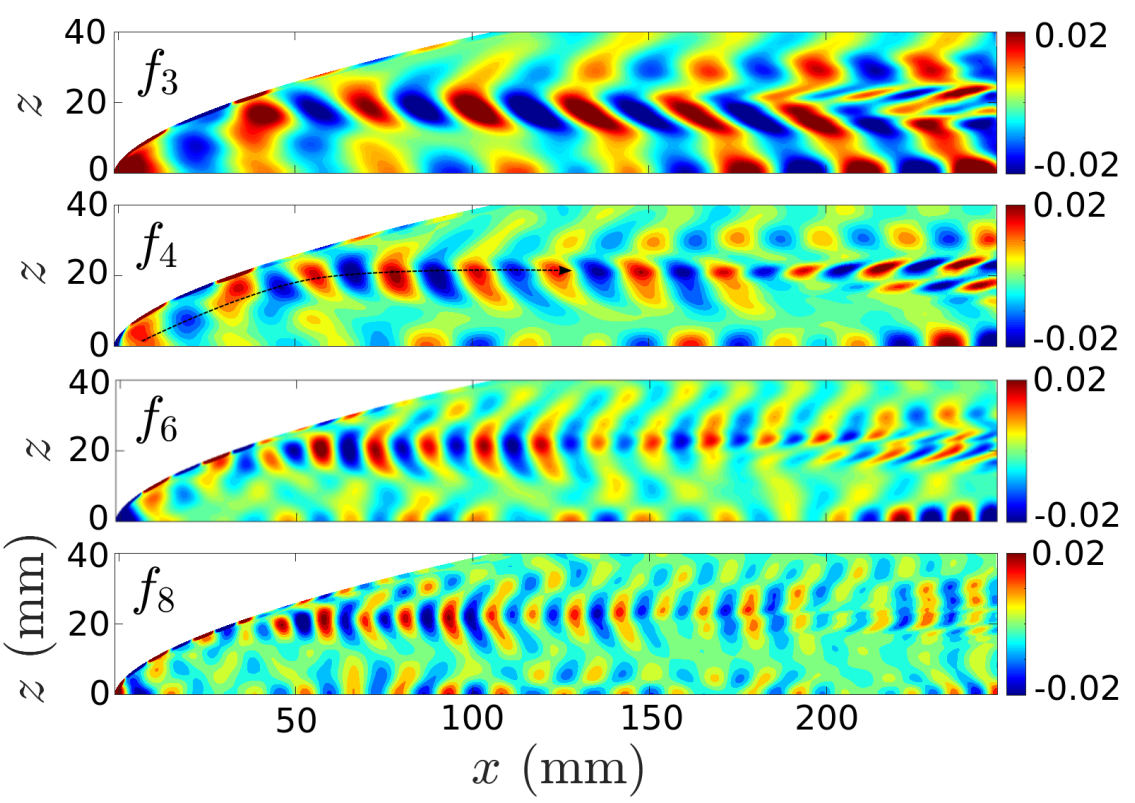

Figure 18: Real part of Fourier-transformed wall-pressure fluctuations $\left(p_{w}^{\prime}\right)$ at different frequencies. Same $x$-axis for all figures

Both lower and higher frequencies show high fluctuations near the leading edge, corresponding to waves travelling in the direction of the flow along the attachment line. Then, at about $z=20$ mm, where sweep angles as high as $\Lambda=70^{\circ}$ are reached, the disturbances detach from the leading edge, and propagate downstream (this path is illustrated by the dashed black line in figure 18 for the frequency $f_{4}$ ). The amplitude of the disturbances released from the leading edge onto the wedge surface reaches very high values 
near the wedge junction, namely between $x=40 \mathrm{~mm}$ and $x=100 \mathrm{~mm}$, which is the zone characterised by high crossflow values (as seen in VII). Then, dependent on the frequencies, the amplitude either decays downstream, or increases leading to the nonlinear growth of a streamwise streak downstream of $x=200$ $\mathrm{mm}$.

As shown in figure 18 the lower frequencies are characterised by higher amplitude levels, and are responsible for the nonlinear growth and bifurcation of the streamwise streak downstream. Moreover, in the region downstream of $x=200 \mathrm{~mm}$ there is also a strong increase in amplitude of the disturbances along the symmetry plane, which is consistent, as seen in Section VIII.A, with the spreading towards the centerline of the nonlinear breakdown process.

Figures 19 and 20 show the downstream evolution and nonlinear growth of the streaks through crosssections of the temperature and the streamwise vorticity at the positions $x=220 \mathrm{~mm}, x=230 \mathrm{~mm}, x=240$ $\mathrm{mm}$. The main streak is initially located at about $z=20 \mathrm{~mm}$, as evident also in figure 15 , As it moves downstream, it increases rapidly in amplitude and spreads across the span, forming secondary vortices. At $x=230 \mathrm{~mm}$ two distinct co-rotating (negative vorticity) vortices appear with different amplitude. At the position $x=240 \mathrm{~mm}$, both vortices show a higher amplitude, and appear shifted along the span. The main vortex is also shifted upwards, providing the pronounced deformation of the boundary-layer edge seen in figure 19. The formation of a pair of counter-rotating secondary vortices is observed at about $z=13$ $\mathrm{mm}$, corresponding to the streak deflection towards the centerline shown in figure 15 , Another much weaker secondary vortex (with negative sign) is seen to start forming at the boundary layer edge alongside the main vortex, at about the position $z=27.5 \mathrm{~mm}$. This fragmentation of the boundary-layer structure corresponds to a breakdown process, which actually started upstream ( $x \approx 140 \mathrm{~mm})$, as seen in figure 17 , where it involved only the upper region of the boundary layer. 

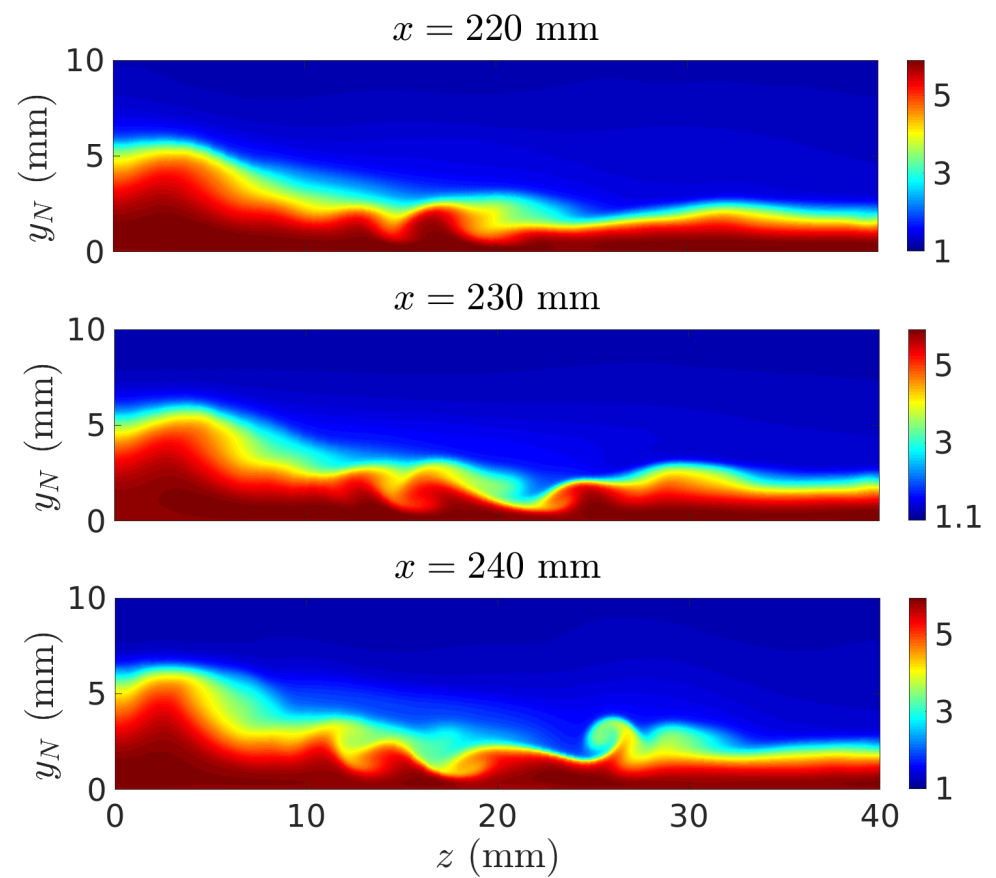

Figure 19: Temperature cross-sections on $z y_{N}$-plane at different $x$-positions. Same $z$-axis for all figures
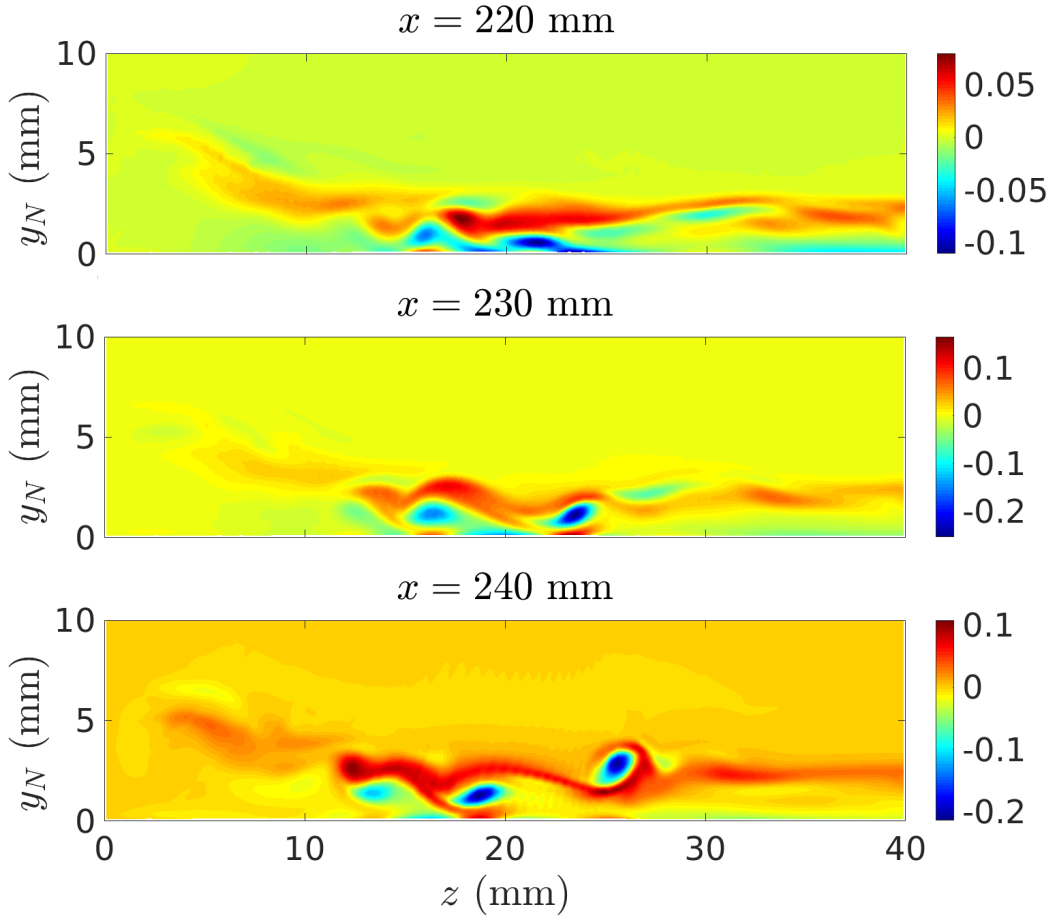

Figure 20: Streamwise vorticity cross-sections on $z y_{N}$-plane at different $x$-positions. Same $z$-axis for all figures

Finally, we present in figure 21 the frequency spectra of the surface pressure fluctuations at the three PCB transducer locations considered in the experiment, namely $x=150 \mathrm{~mm}, x=200 \mathrm{~mm}, x=230 \mathrm{~mm}$, with $z=18 \mathrm{~mm}$ for each position. These locations correspond respectively to the transducers PCB7, PCB8 
and PCB1 used in the experiment [18. It should be mentioned that PCB7 and PCB8 were actually placed at the spanwise position $z=-18 \mathrm{~mm}$ in the experiment, but due to the symmetric flow assumption applied in the simulations, this position corresponds to $z=18 \mathrm{~mm}$ in the present numerical study. This spanwise position corresponds approximately to the position of the main streamwise streak.

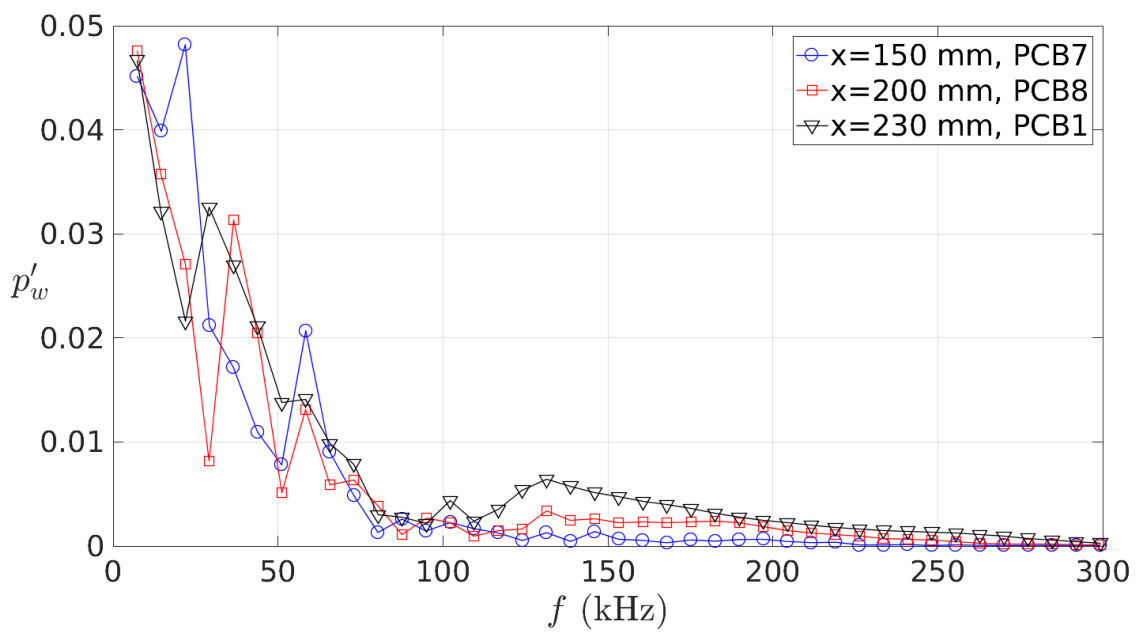

Figure 21: Frequency spectra of the wall pressure fluctuation amplitude at the PCB transducer locations of the experiment

As can be seen in figure 21 the highest pressure levels are concentrated in the frequency band of the imposed freestream disturbances, i.e. $7.3-73 \mathrm{kHz}$. Within this frequency band three local peaks are evident at all the transducer locations: the first is at the lowest frequency, $7.3 \mathrm{kHz}$; the second one varies between the different positions, and is observed at $21.9 \mathrm{kHz}$ at the foremost position (PCB7), $36.5 \mathrm{kHz}$ at the second transducer (PCB8), and at $29.2 \mathrm{kHz}$ at the most downstream position (PCB1); whereas the third (lower) peak is found at $58.4 \mathrm{kHz}$ for all the positions. These frequencies are in a good agreement with the frequencies where traveling crossflow vortices were observed in different studies [28, 31, 34. We also note the presence of a lower peak at a higher frequency, i.e. $131.4 \mathrm{kHz}$, which shows a pronounced growth downstream, reaching its maximum at the transducer located at $230 \mathrm{~mm}$ (PCB1). This is the location where the main streak is observed to split into two separate co-rotating vortices (with reference to figure 20 ) that change their spanwise and vertical position and develop other secondary vortices. This higher frequency is outside the imposed frequency range, thus it has been generated by nonlinear effects, and could be evidence for the start of a secondary instability stage of the breakdown to turbulence.

\section{Conclusion}

Numerical simulations were run to study transition scenarios for 3D fast and slow freestream acoustic wave disturbances, for the MBDA 3D forebody model tested in the Purdue Mach 6 hypersonic wind tunnel. 
The base flow in the leading-edge zone is found to be characterised by a region of inflectional crossflow profiles, extending from the side up to a small distance from the symmetry plane. This was expected, consistent with the high values of the local leading-edge sweep angle. The $3 \mathrm{D}$ effects linked to the presence of the crossflow were found to lead to a reversal of the surface heat flux near the wedge junction, due to very small values of the boundary-layer thickness.

Unsteady numerical simulations with fast and slow acoustic waves were performed at a high freestream amplitude, in order to simulate the noisy wind tunnel conditions of the considered experimental case. Two important differences were observed between the transition patterns in the fast and the slow acoustic wave cases. First, in the fast-wave case the surface heat-flux structure shows signs of $2 \mathrm{D}$ oscillations which resemble a modulation process of the fast mode induced in the boundary layer and the fast acoustic waves, as seen in previous studies. In the slow-wave case, instead, the heat-flux structure is characterised by the formation and downstream growth of streamwise streaks associated with traveling crossflow instabilities originating near the leading edge.

The results with slow acoustic waves provide a better correlation with the experiments, which suggests that the wind tunnel noise was characterised mostly by slow acoustic disturbances during the experimental tests. Transition is induced by the nonlinear growth of streamwise streaks in the off-centerline region. The receptivity study shows that disturbances coming from the nose tip are released through the wedge junction onto the wedge surface $20 \mathrm{~mm}$ off the centerline, and then propagate downstream forming streaks. Frequency spectra of the wall pressure disturbances at different streamwise positions along the main streak show peaks for frequencies between $7.3 \mathrm{kHz}$ and $58.4 \mathrm{kHz}$, consistent with the frequency range of traveling crossflow waves. Higher-frequency disturbances are seen to form downstream, where the main streak undergoes nonlinear growth, indicating that secondary instabilities of the primary crossflow waves may be developing and leading to the breakdown, with generation of secondary vortices spreading towards the centerline. The initial stage of the nonlinear growth is actually observed upstream $(x \approx 140 \mathrm{~mm})$ within the boundary layer. However, in contrast with the experiments, the numerical results show that at this streamwise location the nonlinear breakdown process is confined within the upper part of the boundary layer, thus not yet involving the flow near the wall and the surface heat flux.

The disagreement between the numerical and experimental results in terms of the exact location of the transition point is most probably related to differences between the real freestream noise in the wind tunnel and the disturbances used in the numerical simulations, in terms of both amplitude and frequency content, which would indicate, for example, that even higher noise levels were reached in the wind tunnel runs. This motivates future DNS studies aimed at improving the calibration of the numerical freestream disturbance model to reliably replicate the real experimental noise. 


\section{Acknowledgments}

This work has been carried out in the scope of the NATO STO AVT-240 Hypersonic Boundary-Layer Transition Prediction task group. Computer time on the UK National Supercomputing Service (ARCHER) was provided by the UK Turbulence Consortium (UKTC) under EPSRC (Engineering and Physical Sciences Research Council) Grant No. EP/L000261/1. Finally, support is gratefully acknowledged from EPSRC under the Grant No. EP/J016381/2 'Thermal and Reactive Flow Simulation on High-End Computers' for additional computer time.

\section{References}

[1] Andersson, P., Brandt, L., Bottaro, A., and Henningson, D. S., "On the breakdown of boundary layer streaks", Journal of Fluid Mechanics, 428, February 2001, pp. 29-60.

doi:10.1017/S0022112000002421

[2] Brandt, L., and Henningson, D. S., "Transition of streamwise streaks in zero-pressure-gradient boundary layers", Journal of Fluid Mechanics, 472, December 2002, pp. 229-261.

doi:10.1017/S0022112002002331

[3] Saric, W., Reed, H., and Kerschen, E., "Boundary-layer receptivity to freestream disturbances", Annual Review of Fluid Mechanics, 34(1), 2002, pp. 291-319.

doi:10.1146/annurev.fluid.34.082701.161921

[4] Fedorov, A. V., and Khokhlov, A. P., "Prehistory of Instability in a Hypersonic Boundary Layer", Theoretical and Computational Fluid Dynamics, 14(6), 2001, pp. 359-375.

doi:10.1007/s001620100038

[5] Fedorov, A. V., "Transition and Stability of High-Speed Boundary Layers", Annual Review of Fluid Mechanics, 43(1), 2011, pp. 79-95.

doi:10.1146/annurev-fluid-122109-160750

[6] Zhong, X., and Ma, Y., "Boundary-layer receptivity of Mach 7.99 flow over a blunt cone to free-stream acoustic waves", Journal of Fluid Mechanics, 556, June 2006, pp. 55-103.

doi:10.1017/S0022112006009293

[7] Zhong, X., and Wang, X., "Direct Numerical Simulation on the Receptivity, Instability, and Transition of Hypersonic Boundary Layers", Annual Review of Fluid Mechanics, 44(1), 2012, pp. 527-561. doi:10.1146/annurev-fluid-120710-101208

26 of 31

American Institute of Aeronautics and Astronautics 
[8] Mack, L. M., "Boundary-layer linear stability theory", Special Course on Stability and Transition of Laminar Flow, California Inst. of Technology, Jet Propulsion Lab., AGARD Rept. 709, Pasadena, CA, 1984, pp. 3.1-81.

[9] Stetson, K. F., Thompson, E. R., Donaldson, J. C., and Siler, L. G., "Laminar Boundary Layer Stability Experiments on a Cone at Mach 8. II-Blunt Cone", 22nd Aerospace Sciences Meeting, AIAA Paper 1984$0006,1984$.

[10] Fedorov, A. V., Malmuth, N. D., Rasheed, A., and Hornung, H. G., "Stabilization of hypersonic boundary layers by porous coatings", AIAA Journal, 39(4), 2001, pp. 605-610.

doi: $10.2514 / 2.1382$

[11] Ma, Y., and Zhong, X., "Receptivity of a supersonic boundary layer over a flat plate, Part 3: Effects of different types of free-stream disturbances", Journal of Fluid Mechanics, 532, June 2005, pp. 63-109. doi:10.1017/S0022112005003836

[12] Balakumar, P., "Receptivity of a supersonic boundary layer to acoustic disturbances", AIAA Journal, 47(5), 2009, pp. 1069-1078.

doi:10.2514/1.33395

[13] Malik, M. R., and Balakumar, P., "Acoustic receptivity of Mach 4.5 boundary layer with leading-edge bluntness", Theoretical and Computational Fluid Dynamics, 21(5), 2007, pp. 323-342. doi:10.1007/s00162007-0050-5

[14] Kara, K., Balakumar, P., and Kandil, O. A., "Receptivity of Hypersonic Boundary Layers Due to Acoustic Disturbances over Blunt Cone", 45th AIAA Aerospace Sciences Meeting and Exhibit, AIAA Paper 2007-945, January 2007.

[15] Egorov, I. V., Sudakov, V. G., and Fedorov, A. V., "Numerical modeling of the receptivity of a supersonic boundary layer to acoustic disturbances", Fluid Dynamics, 41(1), 2006, pp. 37-48. doi:10.1007/s10697-006-0020-4

[16] Cerminara, A., and Sandham, N., "Boundary-layer receptivity and breakdown in hypersonic flow over a swept blunt wedge with three-dimensional freestream acoustic disturbances", 8th AIAA Flow Control Conference, AIAA AVIATION Forum, AIAA Paper 2016-4247, 2016.

[17] Cerminara, A., and Sandham, N. D., "Acoustic leading-edge receptivity for supersonic/hypersonic flows over a blunt wedge", AIAA Journal, 2017, pp. 4234-4244.

doi:10.2514/1.J055749

27 of 31

American Institute of Aeronautics and Astronautics 
[18] Durant, A., André, T., Edelman, J. B., Chynoweth, B. C., and Schneider, S. P., "Mach 6 Quiet Tunnel Laminar to Turbulent Investigation of a Generic Hypersonic Forebody", 20th AIAA International Space Planes and Hypersonic Systems and Technologies Conference, AIAA Paper 2015-3575, 2015.

[19] Orlik, E., Fedioun, I., and Lardjane, N., "Hypersonic Boundary-Layer Transition Forced by Wall Injection: A Numerical Study", Journal of Spacecraft and Rockets, 51(4), 2014, pp. 1306-1318. doi:10.2514/1.A32645

[20] André, T., Orlik, E., Durant, A., and Fedioun, I., "Global mode analysis in the L/T transition of a hypersonic boundary layer forced by wall injection", Procedia IUTAM, 14, 2015, pp. 58-67. doi:10.1016/j.piutam.2015.03.024

[21] André, T., Durant, A., and Fedioun, I., "Numerical study of supersonic boundary-layer transition due to sonic wall injection", AIAA Journal, 55(5), 2017, pp. 1530-1547.

doi:10.2514/1.J055164

[22] Saric, William S., Helen L. Reed, and Edward B. White., "Stability and transition of three-dimensional boundary layers", Annual Review of Fluid Mechanics, 35(1), 2003, pp. 413-440.

doi:10.1146/annurev.fluid.35.101101.161045

[23] Reed, H. L., and Saric, W. S., "Stability of three-dimensional boundary layers", Annual Review of Fluid Mechanics, 21(1), 1989, pp. 235-284.

[24] Bippes, H., "Basic experiments on transition in three-dimensional boundary layers dominated by crossflow instability", Progress in Aerospace Sciences, 35(4), 1999, pp. 363-412.

doi:10.1016/S0376-0421(99)00002-0

[25] Kohama, Y., "Some expectation on the mechanism of cross-flow instability in a swept wing flow", Acta Mechanica, 66.1-4, 1987, pp. 21-38.

doi:10.1007/BF01184283

[26] White, Edward B., and William S. Saric., "Secondary instability of crossflow vortices", Journal of Fluid Mechanics, 525, February 2005, pp. 275-308.

doi:10.1017/S002211200400268X

[27] Borg, M. P., Kimmel, R. L., and Stanfield, S., "Traveling crossflow instability for the HIFiRE-5 elliptic cone", Journal of Spacecraft and Rockets, 52(3), 2015, pp. 664-673.

doi:10.2514/1.A33145

28 of 31

American Institute of Aeronautics and Astronautics 
[28] Craig, S. A., and Saric, W. S., "Crossflow instability in a hypersonic boundary layer", Journal of Fluid Mechanics, 808, October 2016, pp. 224-244.

doi:10.1017/jfm.2016.643

[29] Kuehl, J., Perez, E., and Reed, H., "JoKHeR: NPSE simulations of hypersonic crossflow instability", AIAA 2012-0921, 50th AIAA Aerospace Sciences Meeting including the New Horizons Forum and Aerospace Exposition, 2012, Nashville, TN.

doi:10.2514/6.2012-921

[30] Oliviero, N. B., Kocian, T. S., Moyes, A., and Reed, H. L., "EPIC: NPSE analysis of hypersonic crossflow instability on yawed straight circular cone", AIAA 2015-2772, 45th AIAA Fluid Dynamics Conference, 2015, Dallas, TX.

doi: $10.2514 / 6.2015-2772$

[31] Ward, C., Henderson, R., and Schneider, S. P., "Secondary Instability of Stationary Crossflow Vortices on an Inclined Cone at Mach 6", AIAA 2015-2773, 45th AIAA Fluid Dynamics Conference, 2015, Dallas, TX.

doi: $10.2514 / 6.2015-2773$

[32] Choudhari, M. M., Li, F., Chang, C. L., Carpenter, M., Streett, C., Malik, M. R., and Duan, L., "Towards bridging the gaps in holistic transition prediction via numerical simulations", AIAA 2013-2718, 21st AIAA Computational Fluid Dynamics Conference, 2013, San Diego, CA.

doi:10.2514/6.2013-2718

[33] Li, F., Choudhari, M. M., Duan, L., and Chang, C. L., "Nonlinear development and secondary instability of traveling crossflow vortices", Physics of Fluids, 26(6), 2014, pp. 064104-1-19.

doi:10.1063/1.4883256

[34] Li, F., Choudhari, M., Paredes, P., and Duan, L., "High-frequency instabilities of stationary crossflow vortices in a hypersonic boundary layer", Physical Review Fluids, 1(5), 2016, pp. 053603-1-32. doi:10.1103/PhysRevFluids.1.053603

[35] Balakumar, P., and Owens, L., "Stability of hypersonic boundary layers on a cone at an angle of attack", AIAA 2010-4718, 40th Fluid Dynamics Conference and Exhibit, 2010, Chicago, IL. doi: $10.2514 / 6.2010-4718$

[36] Balakumar, P., and King, R. A., "Receptivity and stability of supersonic swept flows", AIAA Journal, 50(7), 2012, pp. 1476-1489.

doi:10.2514/1.J051064 
[37] Bartkowicz, M. D., Subbareddy, P. K., and Candler, G. V., "Simulation of boundary layer transition on elliptic cones in hypersonic flow", AIAA 2010-1064, 48th AIAA Aerospace Sciences Meeting Including the New Horizons Forum and Aerospace Exposition, 2010, Orlando, FL.

doi:10.2514/6.2010-1064

[38] Dinzl, D. J., and Candler, G. V., "Direct Simulation of Hypersonic Crossflow Instability on an Elliptic Cone", AIAA Journal, 55(6), 2017, pp. 1769-1782.

doi:10.2514/1.J055130

[39] Paredes, P., and Theofilis, V., "Centerline instabilities on the hypersonic international flight research experimentation HIFiRE-5 elliptic cone model", Journal of Fluids and Structures, 53, February 2015, pp. $36-49$

doi:10.1016/j.jfluidstructs.2014.11.002

[40] Paredes, P., Grosse R., Theofilis, V., Kimmel, R., "Linear modal instabilities of hypersonic flow over an elliptic cone", Journal of Fluid Mechanics, 804, October 2016, pp. 442-466.

doi:10.1017/jfm.2016.536

[41] Juliano, T. J., Borg, M. P., and Schneider, S. P., "Quiet tunnel measurements of HIFiRE-5 boundarylayer transition", AIAA Journal, 53(4), 2015, pp. 832-846.

doi:10.2514/1.J053189

[42] Schneider, S. P., "Effects of high-speed tunnel noise on laminar-turbulent transition", Journal of Spacecraft and Rockets, 38(3), 2001, pp. 323-333.

doi:10.2514/2.3705

[43] Schneider, S. P., "Development of hypersonic quiet tunnels", Journal of Spacecraft and Rockets, 45(4), 2008, pp. 641-664.

doi:10.2514/1.34489

[44] Schneider, S. P., "Developing mechanism-based methods for estimating hypersonic boundary-layer transition in flight: The role of quiet tunnels", Progress in Aerospace Sciences, 72, January 2015, pp. 17-29. doi:10.1016/j.paerosci.2014.09.008

[45] Masutti, D., Spinosa, E., Chazot, O., and Carbonaro, M., "Disturbance level characterization of a hypersonic blowdown facility", AIAA Journal, 50(12), 2012, pp. 2720-2730. doi:10.2514/1.J051502

[46] Parziale, N. J., Shepherd, J. E., and Hornung, H. G., "Free-stream density perturbations in a reflectedshock tunnel", Experiments in Fluids, 55(2), 2014, pp. 1665-1-10.

30 of 31 
[47] Duan, L., Choudhari, M. M., and Wu, M., "Numerical study of acoustic radiation due to a supersonic turbulent boundary layer", Journal of Fluid Mechanics, 746, May 2014, pp. 165-192.

doi:10.1017/jfm.2014.116

[48] Wagner, A., Schülein, E., Petervari, R., Hannemann, K., Ali, S. R., Cerminara, A., and Sandham, N. D., "Combined free-stream disturbance measurements and receptivity studies in hypersonic wind tunnels by means of a slender wedge probe and direct numerical simulation", Journal of Fluid Mechanics, 842, 2018, pp. 495-531.

doi:10.1017/jfm.2018.132

[49] Cerminara, A., "Boundary-layer receptivity and breakdown mechanisms for hypersonic flow over blunt leading-edge configurations", Doctoral Thesis, University of Southampton Repository, 2017

[50] Yee, H. C., Sandham, N. D., and Djomehri, M. J., "Low-dissipative high-order shock-capturing methods using characteristic-based filters", Journal of Computational Physics, 150(1), 1999, pp. 199-238.

doi:10.1006/jcph.1998.6177

[51] Sandham, N. D., Li, Q., and Yee, H. C., "Entropy splitting for high-order numerical simulation of compressible turbulence", Journal of Computational Physics, 178(2), 2002, pp. 307-322.

doi:10.1006/jcph.2002.7022

[52] De Tullio, N., Paredes, P., Sandham, N. D., and Theofilis, V., "Laminar-turbulent transition induced by a discrete roughness element in a supersonic boundary layer", Journal of Fluid Mechanics, 735, November 2013, pp. 613-646.

doi:10.1017/jfm.2013.520

[53] Cerminara, A., Durant, A., André, T., Sandham, N., and Taylor, N. J., "DNS of Acoustic Receptivity and Breakdown in a Mach 6 Flow over a Generic Forebody", AIAA 2018-0348, AIAA Aerospace Sciences Meeting, 2018, Kissimmee, FL.

doi: $10.2514 / 6.2018-0348$

31 of 31

American Institute of Aeronautics and Astronautics 